\title{
The spectrum of Poincaré recurrence
}

\author{
KA-SING LAU† and LIN SHU†† \\ $\dagger$ Department of Mathematics, The Chinese University of Hong Kong, Shatin, Hong Kong \\ (e-mail:kslau@cuhk.edu.hk) \\ \$ School of Mathematical Sciences, Peking University, Beijing 100871, \\ People's Republic of China \\ (e-mail:lshu@math.pku.edu.cn)
}

(Received 30 September 2007 and accepted in revised form 8 December 2007)

\begin{abstract}
We investigate the relationship between Poincaré recurrence and topological entropy of a dynamical system $(X, f)$. For $0 \leq \alpha \leq \beta \leq \infty$, let $D(\alpha, \beta)$ be the set of $x$ with lower and upper recurrence rates $\alpha$ and $\beta$, respectively. Under the assumptions that the system is not minimal and that the map $f$ is positively expansive and satisfies the specification condition, we show that for any open subset $\emptyset \neq U \subseteq X, D(\alpha, \beta) \cap U$ has the full topological entropy of $X$. This extends a result of Feng and Wu [The Hausdorff dimension of recurrence sets in symbolic spaces. Nonlinearity 14 (2001), 81-85] for symbolic spaces.
\end{abstract}

\section{Introduction}

Let $X$ be a compact metric space and let $f$ be a continuous transformation on $X$. We call the pair $(X, f)$ a dynamical system. The orbit of a point $x \in X$ is the set of iterates of $x$ under $f$, i.e. the sequence $\left\{f^{k}(x)\right\}_{k=0}^{\infty}$. Since $X$ is compact, under some measurepreserving property most orbits are expected to return to the neighborhoods of their starting points. Indeed, the famous Poincaré recurrence theorem says that if $\mu$ is an $f$-invariant probability measure on $X$ and $A$ is a measurable subset with positive measure, then for almost all points $x$ in $A$, the orbit of $x$ will return to $A$ infinitely many times.

To study quantitatively the recurrence behavior of the dynamical system, we define, for any point $x$ in a subset $A$ of $X$, the first (Poincaré) return time of $x$ to $A$ as

$$
\tau_{A}(x)=\inf \left\{i>0: f^{i}(x) \in A\right\} .
$$

Let $\left\{A_{n}\right\}$ be a sequence of sets shrinking to the point $x$; it is natural to consider the exponential rate of increase of the quantity $\tau_{A_{n}}(x)$. In 1993, Ornstein and Weiss [20] first analyzed this rate for a partition under the action of $f$. Let $\xi$ be a finite partition of 
$X$ and let $\xi_{-n}(x)$ be the intersection of all the elements of $\xi, f^{-1}(\xi), \ldots, f^{-n+1}(\xi)$ that contain $x$. It was proved that if $\mu$ is $f$-ergodic, then with probability one,

$$
\lim _{n \rightarrow \infty} \frac{1}{n} \log \tau_{\xi_{-n}(x)}(x)=h_{\mu}(f, \xi),
$$

where $h_{\mu}(f, \xi)$ is the measure-theoretic entropy of $f$ with respect to the partition $\xi$. This remarkable theorem points out that the chaotic behavior of a system, which is described by $h_{\mu}(f, \xi)$, is reflected in the local recurrence rate of the generic orbits. The theorem generated a great deal of interest, and further substantial investigations can be found in the literature. For example, in [26] the partition $\xi_{-n}(x)$ was replaced by balls of radius $r$ centered at $x$; it was shown that if $f$ is a piecewise $C^{1+\alpha}$ monotonic interval map, then for an $f$-ergodic measure $\mu$ with positive entropy,

$$
\lim _{r \rightarrow 0} \frac{\log \tau_{B(x, r)}(x)}{-\log r}=\operatorname{dim}_{H}(\mu) \quad \mu \text {-a.e. }
$$

Other results in this direction can be found in $[2,3]$. Here $\operatorname{dim}_{H}(\mu)$ denotes the Hausdorff dimension of $\mu$, which equals $\lim _{r \rightarrow 0} \log (\mu(B(x, r)) / \log r$ (the local dimension of $\mu$ ) for $\mu$-almost all $x$ (see [8]).

The expression in (1) resembles the local dimension of a measure in the theory of multifractal formalism. Indeed, Feng and $\mathrm{Wu}[\mathbf{1 5}]$ undertook the first investigation of this on the full shift spaces of finite symbols. Let $\Sigma^{\mathbb{N}}$ be the canonical infinite product space of finite symbols with the shift map $S$. Given any Gibbs measure $\mu$ on $\left(\Sigma^{\mathbb{N}}, S\right)$ associated with a Hölder continuous potential, it is well known that for the level sets

$$
K_{\alpha}:=\left\{x \in \Sigma^{\mathbb{N}}:-\lim _{n \rightarrow \infty} \frac{1}{n} \log \mu\left([x]_{n}\right)=\alpha\right\}
$$

(where $[x]_{n}$ is the standard cylinder set), $\operatorname{dim}_{\mathrm{H}}\left(K_{\alpha}\right)$ is (a multiple of) the entropy of a certain Gibbs measure $\mu_{\alpha}$ induced by $\mu$ and the potential [21]; moreover, the dimension spectrum $\Psi(\alpha)=\operatorname{dim}_{\mathrm{H}}\left(K_{\alpha}\right)$ is a strictly concave function. For the local recurrence, it was shown that for any $0 \leq \alpha \leq \beta \leq \infty$,

$$
\operatorname{dim}_{\mathrm{H}}\left\{x \in \Sigma^{\mathbb{N}}: \underset{n \rightarrow \infty}{\lim } \frac{1}{n} \log \tau_{[x]_{n}}(x)=\alpha, \varlimsup_{n \rightarrow \infty} \frac{1}{n} \log \tau_{[x]_{n}}(x)=\beta\right\}=\operatorname{dim}_{\mathrm{H}}\left(\Sigma^{\mathbb{N}}\right) .
$$

This unusual conclusion is significantly different from the local dimensions, as the dimension spectrum is a constant. The result was also extended in [27] to conformal repellers, and in [19] to self-conformal sets satisfying the strong open set condition.

In this paper, we will consider recurrence by making use of the Bowen metric on the dynamical system. Recall that the $n$th Bowen metric on $(X, f)$ is defined by

$$
d_{n}(x, y):=\max \left\{d\left(f^{i}(x), f^{i}(y)\right): i=0,1, \ldots, n-1\right\} \text { for all } x, y \in X .
$$

This is a basic notion in the study of topological entropy $[\mathbf{6 , 2 1 , 3 2 ] . ~ I t ~ i s ~ a l s o ~ u s e f u l ~ i n ~}$ connection with measure-theoretic entropy $h_{\mu}(f)$ [7, 16]; in [7], Brin and Katok showed that if $\mu$ is an $f$-ergodic measure on $X$, then

$$
h_{\mu}(f)=-\lim _{\epsilon \rightarrow 0} \underset{n \rightarrow \infty}{\lim } \frac{1}{n} \log \mu\left(B_{n}(x, \epsilon)\right) \quad \mu \text {-a.e., }
$$


where $B_{n}(x, \epsilon)$ is the $n$th Bowen ball of radius $\epsilon$ centered at $x$. We define the local (Poincaré) recurrence rates $\underline{\tau}(x)$ and $\bar{\tau}(x)$ by

$$
\lim _{\epsilon \rightarrow 0} \lim _{n \rightarrow \infty} \frac{1}{n} \log \tau_{B_{n}(x, \epsilon)}(x) \text { and } \lim _{\epsilon \rightarrow 0} \varlimsup_{n \rightarrow \infty} \frac{1}{n} \log \tau_{B_{n}(x, \epsilon)}(x),
$$

respectively. Based on the results by Ornstein and Weiss [20] and Katok [16], in [29] one of the authors proved that for any $f$-invariant probability measure $\mu$ on $X$ with $h_{\mu}(f)<\infty$,

$$
\bar{\tau}(x)=\underline{\tau}(x)=h_{\mu}(f, x) \quad \mu \text {-a.e., }
$$

where $h_{\mu}(f, x)\left(=-\lim _{\epsilon \rightarrow 0} \underline{\lim }_{n \rightarrow \infty}(1 / n) \log \mu\left(B_{n}(x, \epsilon)\right)\right.$ a.e. $)$ is the local entropy of $\mu$ at $x$. (See also [10] for a different approach to proving the same result.) This reveals the connection between the local recurrence rates and the entropy of the measure in terms of the Bowen metric.

Our goal in this paper is to consider the spectrum structure due to the recurrence, as in (2); our set-up for the recurrence is to use Bowen balls on the dynamical systems and to consider the topological entropy. We assume that the map $f$ is positively expansive and satisfies the specification condition (SPEC). The exact definitions are given in $\$ 2$ and $\S 3$. Roughly speaking, a map $f$ is positively expansive if it increases the distance between any two distinct points in an iteration; $f$ has the SPEC if one can always find a single orbit to interpolate between different pieces of orbits, up to a pre-assigned error. There are well-known examples of dynamical systems with the SPEC-for example, the canonical symbolic space with the shift map or, more generally, the mixing subshifts of finite type [9]. There are also some expanding dynamical systems that are factors of subshifts of finite type [25] and hence have the SPEC if the systems are mixing [30] (e.g. the mixing conformal repellers). On the other hand, there are dynamical systems with the SPEC that cannot be embedded into a symbolic space. We shall discuss this in more detail in $\S 3$.

For $0 \leq \alpha \leq \beta \leq \infty$, let

$$
D(\alpha, \beta)=\{x \in X: \underline{\tau}(x)=\alpha, \bar{\tau}(x)=\beta\}
$$

be level sets for Poincaré recurrence, and let $h_{t}(f, A)$ be the topological entropy of a subset $A \subseteq X$. Our main theorem is the following.

THEOREM 1.1. Let $(X, f)$ be a non-minimal dynamical system which is positively expansive and satisfies the specification condition. Then, for any $0 \leq \alpha \leq \beta \leq \infty$ and for any non-empty open set $U$ of $X$, we have

$$
h_{t}(f, U \cap D(\alpha, \beta))=h_{t}(f, X) .
$$

By a non-minimal dynamical system, we mean ${\overline{\left\{f^{k}(x)\right.}}_{k} \neq X$ for some $x \in X$. The theorem says that $D(\alpha, \beta)$ always has the full topological entropy $h_{t}(f, X)$ (as in (2)). It differs from the multifractal theory for local entropy of a measure $\mu$. In some sense this is attributable to the fact that for a given Gibbs measure $\mu$ associated with a potential $\varphi$, the condition $h_{\mu}(f, x)=\alpha$ is equivalent to $\lim _{n \rightarrow \infty}(1 / n) \sum_{i=0}^{n-1} \varphi\left(f^{i+j}(x)\right)=\alpha^{\prime}$ (for some $\alpha^{\prime}$ ) for all $j$; this places a restriction on the collection of possible pieces of orbits $\left\{f^{j}(x), \ldots, f^{j+n-1}(x)\right\}$ for all $j \in \mathbb{N}$, whereas $\underline{\tau}(x)=\alpha$ (and also $\bar{\tau}(x)=\beta$ ) imposes a 
much weaker restriction on those pieces. We will see later that such limits may only depend on the orbit of $x$ in a sequence of positions with 'zero density' in $\mathbb{N}$ (see Propositions 5.2 and 5.4). Hence the freedom of choosing orbits in other places implies that the set $D(\alpha, \beta)$ is as large as $X$ in the sense of topological entropy.

The proof of the theorem relies on constructing sufficiently many $x$ that have the desired recurrence rates $\alpha$ and $\beta$. The main idea, inspired by $[\mathbf{1 3}, \mathbf{3 1}]$, is to use the Moran fractal. A Moran fractal is a Cantor-type set defined by iteration; it has a tractable structure and has been used extensively for estimating entropies and dimensions (see e.g. $[1,11,17,18,21,22])$. In our context, we will make use of a special dynamically defined (by the Bowen metric) Moran fractal (Definition 4.1). There are two major steps in the construction; in the first step, we prove the following.

Proposition 1.2. Suppose $(X, f)$ is a positively expansive dynamical system which satisfies the SPEC. Then there exists a dynamically defined Moran fractal $F \subset X$ such that $h_{t}(f, F)=h_{t}(f, X)$.

This proposition is proved via Theorem 4.3 and Propositions 4.4 and 4.6. Note that in this step there is no restriction on recurrence rates for the elements in $F$. Our next step, actually the more elaborate step, is to modify the elements $y \in F$ to form another Moran fractal $F^{\prime}$ having approximately the same entropy, while at the same time each $x \in F^{\prime}$ has the required recurrence rates. The technique is to keep adding the previous segment inductively to an appropriate position in the later part of the orbit sequence of $y$ to ensure recurrence, and meanwhile use the SPEC to 'shadow' the sequence by another approximating orbit sequence of $x$.

We organize the paper as follows. In $\S 2$ we define topological entropy and positively expansive maps, and present some preliminary properties. We introduce the specification condition in $\S 3$, and set up the shadowing maps of the orbits. Proposition 1.2 is then proved in $\S 4$, and the proof of Theorem 1.1 is in $\S 5$. The insertion of the segments into the orbit sequences of $F$ to obtain the prescribed recurrence rates is a rather complicated procedure; we divide the proof into three subsections to clarify the construction of $D(\alpha, \beta)$. Some of the technical lemmas are deferred to Appendix A.

\section{Preliminaries}

Throughout this paper we assume that $X$ is a compact metric space and that $f: X \rightarrow X$ is a continuous map. We call such $(X, f)$ a dynamical system and we let $d_{n}$ denote the Bowen metric as defined in (3). We adopt the following definition of topological entropy [21].

Definition 2.1. Let $(X, f)$ be a dynamical system. Given any subset $Z \subseteq X$, we define, for $\epsilon>0, s>0$ and $N>0$,

$$
m(Z ; s, N, \epsilon)=\inf _{\Gamma} \sum_{i} e^{-s n_{i}}
$$

where $\Gamma=\left\{B_{n_{i}}\left(x_{i}, \epsilon\right)\right\}_{i}$ is any collection of $n_{i}$ th Bowen balls, with $\min _{i} n_{i}>N$, that covers $Z$. Let

$$
m(Z ; s, \epsilon)=\lim _{N \rightarrow \infty} m(Z ; s, N, \epsilon)=\sup _{N>0} m(Z ; s, N, \epsilon) .
$$


The critical value $h_{t}(f, Z ; \epsilon)$ is defined as

$$
m(Z ; s, \epsilon)= \begin{cases}+\infty, & s<h_{t}(f, Z ; \epsilon), \\ 0, & s>h_{t}(f, Z ; \epsilon) .\end{cases}
$$

The topological entropy of $Z$ is then defined as $h_{t}(f, Z)=\lim _{\epsilon \rightarrow 0} h_{t}(f, Z ; \epsilon)$. In particular, if $Z=X$, then we write $h_{t}(f)$ for simplicity.

The quantity $m(Z ; s, N, \epsilon)$ is non-decreasing with respect to $N$; hence the limit $m(Z ; s, \epsilon)$ exists. The critical value $h_{t}(f, Z ; \epsilon)$ is non-increasing with respect to $\epsilon$, so the limit as $\epsilon \rightarrow 0$ exists and the notion of topological entropy is well defined.

We say two metrics $d$ and $d^{\prime}$ on $X$ are uniformly (topologically) equivalent if the identity maps are uniformly continuous with respect to the two metrics. It is easy to show that the topological entropy $h_{t}(f, Z)$ and the recurrence exponents $\underline{\tau}(\cdot), \bar{\tau}(\cdot)$ are independent of the uniformly equivalent metric used.

Definition 2.2. Let $(X, f)$ be a dynamical system. The map $f$ is said to be positively expansive if there exists $\gamma>0$ such that for any $x \neq y \in X$,

$$
d\left(f^{n}(x), f^{n}(y)\right)>\gamma \quad \text { for some } n>0 .
$$

In this case, we call $(X, f)$ a positively expansive system. (In what follows we will fix the expansive constant $\gamma$.)

This class of maps was introduced by Williams [33] and Eisenberg [12]. It is known [23] that for a compact metric space $X, f: X \rightarrow X$ being positively expansive is equivalent to the existence of a compatible metric $d^{\prime}$ on $X$ and constants $\eta>0, \lambda>1$ such that

$$
d^{\prime}(f(x), f(y)) \geq \lambda d^{\prime}(x, y) \text { for all } d^{\prime}(x, y)<\eta .
$$

For any finite cover $\mathcal{C}$ of $X$, define $\operatorname{diam} \mathcal{C}$ to be the maximum of the diameters of the members of $\mathcal{C}$. Also let $\mathcal{C}_{-n}=\bigvee_{i=0}^{n-1} f^{-i}(\mathcal{C})$ be the family of intersections of members of $f^{-i}(\mathcal{C})$. Using the same proof as [32, Theorem 5.23] for expansive homeomorphisms, we have the following.

LEMMA 2.3. Let $(X, f)$ be a positively expansive system. For any finite cover $\mathcal{C}$ of $X$ with $\operatorname{diam} \mathcal{C} \leq \gamma, \lim _{n \rightarrow \infty} \operatorname{diam} \mathcal{C}_{-n}=0$.

LeMmA 2.4. Let $(X, f)$ be a positively expansive system. Then for any $\delta<\gamma / 4$ and any $\epsilon>0$, there exists $N>0$ (which depends on $\delta, \epsilon$ ) such that

$$
d_{n+N}(x, y) \leq \delta \Rightarrow d_{n}(x, y)<\epsilon \text { for all } n>0 .
$$

Proof. This lemma has appeared elsewhere in the literature, but we provide a proof here for the reader's convenience.

Choose $x_{1}, \ldots, x_{k}$ such that $\left\{B\left(x_{i}, \gamma / 2-2 \delta\right)\right\}_{i=1}^{k}$ is a cover of $X$. Let $\mathcal{C}=\left\{B\left(x_{i}, \gamma / 2\right): 1 \leq i \leq k\right\}$; this is also a finite cover of $X$, with diam $\mathcal{C} \leq \gamma$. Hence, by Lemma 2.3 , there is $N>0$ such that $\operatorname{diam} \mathcal{C}_{-N}<\epsilon$. Note that $\mathcal{C}$ has Lebesgue number $2 \delta$. (To see this, take $x \in B\left(x_{i}, \gamma / 2-2 \delta\right)$ for some $i \leq k$; then $d(x, y)<2 \delta$ gives $d\left(y, x_{i}\right)<\gamma / 2$. Hence $x, y \in B\left(x_{i}, \gamma / 2\right)$.) Now $d_{n+N}(x, y) \leq \delta$ implies $f^{i} x$ and $f^{i} y$ belong to the same element of $\mathcal{C}$ for $0 \leq i \leq n+N-1$. Consequently $f^{i}(x)$ and $f^{i}(y)$ belong to the same element of $\mathcal{C}_{-N}$ for $0 \leq i \leq n-1$, and $d_{n}(x, y)<\operatorname{diam} \mathcal{C}_{-N}<\epsilon$. 
A set $E \subseteq X$ is called $(n, \epsilon)$-separated if for any $x \neq y$ in $E, d_{n}(x, y) \geq \epsilon . E$ is called maximal if it attains the maximal cardinality. We use $s_{n}(\epsilon)$ to denote the cardinality of a maximal $(n, \epsilon)$-separated set in $X$. It is known [32, Theorem 7.9] that for any continuous map $f$ on $X$,

$$
h_{t}(f)=\lim _{\epsilon \rightarrow \infty} \underset{n \rightarrow \infty}{\lim } \frac{1}{n} \log s_{n}(\epsilon)=\lim _{\epsilon \rightarrow \infty} \varlimsup_{n \rightarrow \infty} \frac{1}{n} \log s_{n}(\epsilon) .
$$

THEOREM 2.5. Let $(X, f)$ be a positively expansive system. Then for any $\delta<\gamma / 4$,

$$
h_{t}(f)=\lim _{n \rightarrow \infty} \frac{1}{n} \log s_{n}(\delta) .
$$

Proof. Let $0<\epsilon<\delta$ and let $N$ be such that (7) holds. Then we have

$$
s_{n+N}(\delta) \geq s_{n}(\epsilon) \geq s_{n}(\delta),
$$

and the conclusion follows from (8).

To conclude this section, we show that the recurrence exponents $\underline{\tau}(\cdot), \bar{\tau}(\cdot)$ defined in (4) can be simplified if we assume the positively expansive property on $f$. Moreover, we can put it in a slightly more general form which will be used later (in Proposition 5.2 and Lemma 5.7).

Proposition 2.6. Let $(X, f)$ be a positively expansive system and let $\gamma$ be an associated expansive constant. Let $\left\{p_{n}\right\}_{n=0}^{\infty}$ be a strictly increasing sequence such that $\lim _{n \rightarrow \infty} p_{n+1} / p_{n}=1$. Then for any $\delta<\gamma / 4$, we have

$$
\underline{\tau}(x)=\varliminf_{n \rightarrow \infty} \frac{1}{p_{n}} \log \tau_{B_{p_{n}}(x, \delta)}(x),
$$

and similarly for $\bar{\tau}(x)$.

Proof. It suffices to consider the case $p_{n}=n$. The general case follows by observing that for any sequence $\left\{a_{n}\right\}_{n=0}^{\infty}$ in $\mathbb{R}, \underline{\lim }_{n \rightarrow \infty} a_{n} / n=\underline{\lim }_{n \rightarrow \infty} a_{p_{n}} / p_{n}$ if $\lim _{n \rightarrow \infty} p_{n+1} / p_{n}=1$ and $\lim _{n \rightarrow \infty} p_{n}=\infty$.

Let $0<\epsilon<\delta$ and let $N$ be such that (7) holds. Then we have $B_{n+N}(x, \delta) \subseteq B_{n}(x, \epsilon) \subseteq$ $B_{n}(x, \delta)$, and so

$$
\tau_{B_{n+N}(x, \delta)}(x) \geq \tau_{B_{n}(x, \epsilon)}(x) \geq \tau_{B_{n}(x, \delta)}(x) .
$$

The desired equality follows from the definition of $\underline{\tau}(\cdot), \bar{\tau}(\cdot)$.

COROLlary 2.7. Let $\Sigma^{\mathbb{N}}$ be the canonical infinite product space of finite symbols with the shift map $S$. Let $\left\{p_{n}\right\}_{n=0}^{\infty}$ be a strictly increasing sequence such that $\lim _{n \rightarrow \infty} p_{n+1} / p_{n}=1$. Then

$$
\underline{\tau}(x)=\varliminf_{n \rightarrow \infty} \frac{1}{p_{n}} \log \tau_{[x]_{p_{n}}}(x),
$$

and similarly for $\bar{\tau}(x)$. (Here $[x]_{n}$ is the cylinder set determined by the first $n$ coordinates of $x$.)

The proof follows easily upon noticing that for given $\epsilon>0, B_{n}(x, \epsilon)$ coincides with $[x]_{n+N}$ for some $N$, and then applying the above proposition. 


\section{The specification condition}

The notion of specification, introduced by Bowen [5], says that one can always find a single orbit to interpolate between different pieces of orbits.

Definition 3.1. Let $(X, f)$ be a dynamical system; $f$ is said to satisfy the condition of specification (SPEC) if for any $\epsilon>0$, there exists an integer $m(\epsilon)$ such that for arbitrary finite intervals of integers $I_{j}=\left[a_{j}, a_{j}+n_{j}-1\right] \cap \mathbb{N}, 0 \leq j<k$, with

$$
\operatorname{dist}\left(I_{i}, I_{j}\right)>m(\epsilon) \text { for all } i \neq j
$$

and for any $y_{0}, \ldots, y_{k-1}$ in $X$, there exists a point $x \in X$ satisfying

$$
d\left(f^{p}(x), f^{p-a_{j}}\left(y_{j}\right)\right)<\epsilon \text { for all } p \in I_{j} \text { and } 0 \leq j<k .
$$

(We follow the tradition not requiring $x$ to be periodic, which is slightly different from the original definition in [5].) For $y \in X$, we let $\left.y\right|_{l}=\left\{y, \ldots, f^{l-1}(y)\right\}$ denote the orbit of $y$ of length $\ell$. In the case where $\operatorname{dist}\left(I_{i}, I_{j}\right)=m+1$, a point $x$ satisfying (9) is said to $\epsilon$-shadow the pieces $\left.y_{0}\right|_{n_{0}}, \ldots,\left.y_{k-1}\right|_{n_{k-1}}$ (with gaps $m(\epsilon)$ ). The constant $m(\epsilon)$ can be interpreted as the time for switching over from one orbit to the other, up to an approximation of $\epsilon$; we fix $m(\epsilon)$ for future use.

There are important classes of positively expansive maps that satisfy the SPEC. The most basic example is the shift map on the full symbolic space; another example is $X$, a subshift space of finite type with shift map mixing on $X$ [9]. A map $f$ on $X$ is said to be (Ruelle) expanding if it is open and positively expansive ([25, p. 143]; see also [24, pp. 3-4] for examples of positively expansive maps that are not open). An expanding map has Markov partitions of arbitrarily small size; hence the system is a factor of a subshift of finite type [25], and has the SPEC if the system is mixing. It follows that topologically mixing conformal repellers (see e.g. [21]) also satisfy the SPEC. The requirement of existence of a finite Markov partition is stronger than the SPEC. Indeed, consider the one-parameter transformations $\left\{T_{\beta}\right\}_{\beta>1}$ on $[0,1]$ defined by taking $T_{\beta}(x)=\beta x(\bmod 1)$. Clearly each map is positively expansive. The following results are known for $T_{\beta}[\mathbf{4 , 2 8}]$ : (i) the set of $\beta$ such that $T_{\beta}$ admits a finite Markov partition is at most countable; and (ii) the set of $\beta$ such that $T_{\beta}$ satisfies the SPEC has Hausdorff dimension 1 and Lebesgue measure 0 .

The following orbit specification lemma [30] (stated in a slightly different way in our context) provides a method of finding a single orbit to approximate an infinite sequence $\left\{y_{i}\right\}_{i=0}^{\infty}$ simultaneously.

LEMMA 3.2. Let $(X, f)$ be a dynamical system that satisfies the SPEC. Let $\left\{\epsilon_{k}\right\}_{k=0}^{\infty} \searrow 0$ be a sequence of positive numbers, and let $m_{k}=m\left(\epsilon_{k}-\epsilon_{k+1}\right)$. Then for any increasing sequence of disjoint finite intervals of integers $I_{k}=\left[a_{k}, a_{k}+n_{k}-1\right]$, with $a_{k+1}-\left(a_{k}+\right.$ $\left.n_{k}\right) \geq m_{k}$, and any sequence $\left\{y_{k}\right\}_{k=0}^{\infty} \in X$, there exists $x \in X$ such that

$$
d\left(f^{j}(x), f^{j-a_{k}}\left(y_{k}\right)\right) \leq \epsilon_{k} \quad \text { for } j \in I_{k}, k \in \mathbb{N} .
$$

(In the case $a_{0}=0$ and $a_{k+1}-\left(a_{k}+n_{k}\right)=m_{k}$, a point $x$ satisfying (10) is said to $\left\{\epsilon_{k}\right\}$ shadow the sequence of pieces $\left\{\left.y_{k}\right|_{n_{k}}\right\}_{k=0}^{\infty}$ with gaps $\left\{m_{k}\right\}_{k=0}^{\infty}$.) 


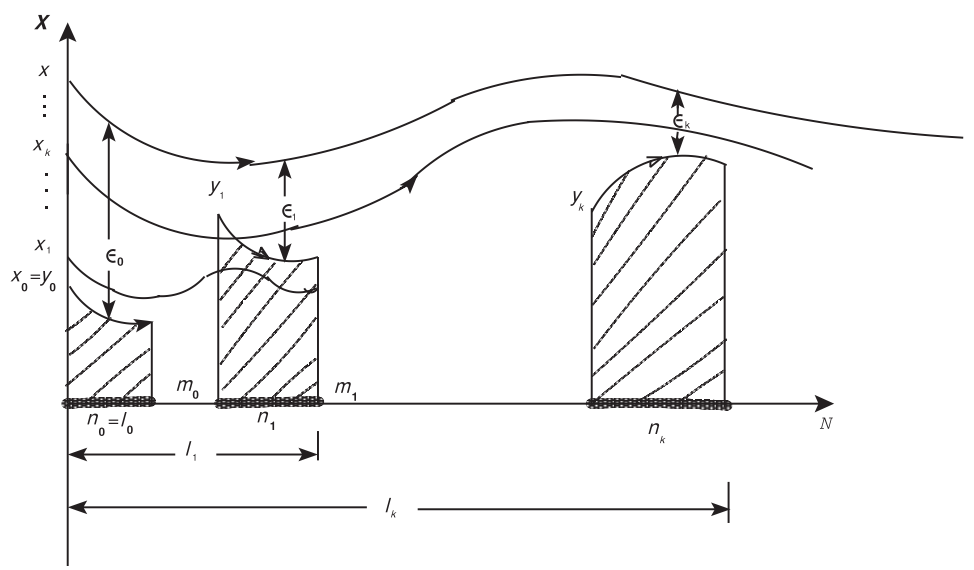

FIGURE 1. Approximation by means of the orbit specification lemma.

Proof. The construction is given in [30] for a special case; we include a proof for completeness. Without loss of generality, assume that $a_{0}=0$. Set $l_{k}=a_{k}+n_{k}$, $\epsilon_{k}^{\prime}=\epsilon_{k}-\epsilon_{k+1}$ and $m_{k}:=m\left(\epsilon_{k}^{\prime}\right)$ for $k \in \mathbb{N}$. We define a sequence $x_{k} \in X$ by induction (see Figure 1). Let $x_{0}=y_{0}$. Since $a_{1}-n_{0} \geq m_{0}$, by the definition of SPEC there exists $x_{1} \in X$ such that

$$
d_{l_{0}}\left(x_{1}, x_{0}\right)<\epsilon_{0}^{\prime} \quad \text { and } \quad d_{n_{1}}\left(f^{a_{1}}\left(x_{1}\right), y_{1}\right)<\epsilon_{0}^{\prime} .
$$

Suppose we have already constructed $x_{k}$. Let $x_{k+1}$ be such that

$$
d_{l_{k}}\left(x_{k+1}, x_{k}\right)<\epsilon_{k}^{\prime} \text { and } d_{n_{k+1}}\left(f^{a_{k+1}}\left(x_{k+1}\right), y_{k+1}\right)<\epsilon_{k}^{\prime} .
$$

Such a point exists due to the SPEC. The sequence $\left\{x_{k}\right\}_{k=0}^{\infty}$ forms a Cauchy sequence, as

$$
d\left(x_{k}, x_{k+1}\right)<d_{l_{k}}\left(x_{k}, x_{k+1}\right)<\epsilon_{k}^{\prime}
$$

and $\sum_{k=0}^{\infty} \epsilon_{k}^{\prime}=\epsilon_{0}<\infty$. Let $x$ be the limit point of $\left\{x_{k}\right\}_{k=0}^{\infty}$. It then follows from (11) that

$$
d\left(f^{j}\left(x_{k}\right), f^{j-a_{n}}\left(y_{n}\right)\right)<\sum_{i=n}^{k} \epsilon_{i}^{\prime}<\epsilon_{n} \quad \text { for } j \in I_{n}, k \geq n .
$$

The conclusion for $x$ follows from taking limits with respect to $k$.

It is clear that the above construction implies the next result.

COROLlary 3.3. Let $\left\{x_{i}\right\}_{i=0}^{\infty}$ and $\left\{l_{i}\right\}_{i=0}^{\infty}$ be as in the proof of Lemma 3.2. Then $x_{i}$ satisfies (11) with respect to $\left\{y_{k}\right\}_{k \leq i}$, and the sequence $\left\{\overline{B_{l_{i}}}\left(x_{i}, 2 \epsilon_{i}\right)\right\}_{i=0}^{\infty}$ decreases to $x$.

We will generalize the orbit specification lemma a little, and introduce some notation to be used in the rest of the paper. Let $\mathcal{X}$ be the collection of initial segments of the orbits of $x \in X$, i.e.

$$
\mathcal{X}=\left\{\left.x\right|_{n}: x \in X, n \geq 1\right\} .
$$

Let $\mathcal{X}^{N}$ be the $N$-tuples of elements in $\mathcal{X}$.

\section{CAmbridge JDUNALS}


(i) Fix $\epsilon>0$ and let $m(\epsilon)$ be as in the definition of SPEC. Suppose that $\left(\left.y_{0}\right|_{n_{0}}, \ldots,\left.y_{N-1}\right|_{n_{N-1}}\right) \in \mathcal{X}^{N}$ is given, and take $x$ to be a point which $\epsilon$-shadows $\left\{y_{k}\right\}_{k<N}$ with gaps $m(\epsilon)$ (note that there may be more than one $x$ satisfying the requirement; we fix one of these choices). Let $l=\sum_{k=0}^{N-1} n_{k}+(N-1) m(\epsilon)$, and define $\Phi_{\epsilon}$ on $\mathcal{X}^{N}$ by

$$
\Phi_{\epsilon}\left(\left.y_{0}\right|_{n_{0}}, \ldots,\left.y_{N-1}\right|_{n_{N-1}}\right)=\left.x\right|_{l} .
$$

Intuitively, $\Phi_{\epsilon}$ glues the orbits together with error bound $\epsilon$ and gaps of length $m(\epsilon)$.

(ii) More generally, for $\left\{\epsilon_{k}\right\}_{k=0}^{\infty} \searrow 0$, let $\epsilon_{k}^{\prime}=\epsilon_{k}-\epsilon_{k+1}$ and let $m_{k}=m\left(\epsilon_{k}^{\prime}\right)$ be as in Definition 3.1. Given $\left(\left.y_{0}\right|_{n_{0}}, \ldots,\left.y_{N-1}\right|_{n_{N-1}}, \ldots\right) \in \mathcal{X}^{\mathbb{N}}$, take $x$ to be a point as in Lemma 3.2 which $\left\{\epsilon_{k}\right\}$-shadows the sequence $\left\{\left.y_{k}\right|_{n_{k}}\right\}_{k=0}^{\infty}$ with gaps $m_{k}$. We then define

$$
\Phi_{\left\{\epsilon_{k}\right\}}\left(\left.y_{0}\right|_{n_{0}}, \ldots,\left.y_{N-1}\right|_{n_{N-1}}, \ldots\right)=x .
$$

In this way, $\Phi_{\left\{\epsilon_{k}\right\}}(\cdot)$ gives a point whose orbit shadows the given sequence of finite orbits with accuracies $\left\{\epsilon_{k}\right\}_{k=0}^{\infty}$ and gaps $\left\{m_{k}\right\}_{k=0}^{\infty}$. For later use, we write $\Phi_{\left\{\epsilon_{k}\right\}}\left(y_{0}\left|n_{0}, \ldots, y_{N}\right|_{n_{N}}\right)$ for the point $x_{N}$ obtained as in Lemma 3.2 for the selection of $x$.

(iii) Let $\left\{z_{j}\right\}_{j=0}^{\infty} \in \mathcal{X}^{\mathbb{N}}$ be a sequence of orbit segments. We group the sequence into blocks as $\left\{z_{\mathbf{j}_{k}}\right\}_{k=0}^{\infty}$ where $z_{\mathbf{j}_{0}}=\left\{z_{0}, \ldots, z_{j_{0}}\right\}, z_{\mathbf{j}_{1}}=\left\{z_{j_{0}+1}, \ldots, z_{j_{1}}\right\}$ and so on. We denote the new sequence by $\mathbf{z}$ for convenience. Let $x=\Phi(\mathbf{z})$ be chosen by first setting $y_{k}=\Phi_{\epsilon}\left(z_{\mathbf{j}_{k}}\right)$ as in (i), then $\Phi_{\left\{\epsilon_{k}\right\}}\left(y_{0}, y_{1}, \ldots\right)$ as in (ii). We see that the orbit of $x$ is within $\epsilon+\epsilon_{0}$ distance of the $\left\{z_{j}\right\}_{j}$ in corresponding places.

In order to ease the notation for later on, we introduce two 'fictitious' symbols $\left\{\bowtie_{k}\right\}_{k=0}^{\infty}$ and $\smile$, and write the block sequence as

$$
\mathbf{z}=z_{0} \smile \cdots \smile z_{j_{0}} \bowtie_{0} z_{j_{0}+1} \smile \cdots \smile z_{j_{1}} \bowtie_{1} \cdots
$$

where the $z_{j}$ connected by $\smile$ means that we will $\epsilon$-shadow each such block by (i), and the $\left\{\bowtie_{k}\right\}_{k}$ means that we will $\left\{\epsilon_{k}\right\}$-shadow the resulting sequence. We also assign a length $m(\epsilon)$ for $\smile$ and a length $m_{k}$ for $\bowtie_{k}$ with respect to $\epsilon_{k}^{\prime}=\epsilon_{k}-\epsilon_{k+1}$. For the $\left\{\epsilon_{k}^{\prime}\right\}$ with a certain convergence rate, we can estimate the length of $m_{k}$ (see Lemma 4.5).

\section{Dynamically defined Moran fractals}

In this section we introduce a dynamically defined Moran fractal, which is a Cantor-type set (cf. [21, Ch. 5]). The entropy of this set is trackable, and our main purpose is to use it to obtain the lower bound of the topological entropy $h_{t}(f, \cdot)$. The fractal we define is a generalization of the constructions used in $[\mathbf{1 3}, \mathbf{1 4}]$ for symbolic spaces and in $[\mathbf{3 1}]$ for dynamical systems satisfying the SPEC.

Let $Q$ be a subset of $\mathbb{N}^{\infty}$. A word $i_{0} \ldots i_{n}$ is called $Q$-admissible if $i_{0} \ldots i_{n} i_{n+1} \ldots$ belongs to $Q$ for some $i_{n+1} i_{n+2} \ldots$ Let $Q_{n}$ denote the $Q$-admissible words of length $n+1$. We assume that $\# Q_{n}$ is finite for each $n$.

Definition 4.1. (cf. [21, Ch. 5]) Let $(X, d)$ be a compact metric space, and let $f$ be a continuous transformation on $X$. A dynamically defined Moran fractal $F$ of $X$ modeled by $Q$ is defined by

$$
F=\bigcap_{n=0}^{\infty} \bigcup_{\substack{\left(i_{0} \ldots i_{n}\right) \\ Q \text {-admissible }}} \Delta_{i_{0} \ldots i_{n}}
$$


where the $\Delta_{i_{0} \ldots i_{n}}$ are closed subsets (called basic sets) in the $n$th level which satisfy:

(C1) $\Delta_{i_{0} \ldots i_{n} j} \subseteq \Delta_{i_{0} \ldots i_{n}}$ for all $i_{0} \ldots i_{n} j$ that are $Q$-admissible;

(C2) $\lim _{n \rightarrow \infty} \operatorname{diam} \Delta_{i_{0} \ldots i_{n}}=0$;

(C3) (dynamical separation condition) there exist $\delta>0$ and $\left\{l_{n}\right\}_{n=0}^{\infty} \uparrow \infty$ such that, for large $n, d_{l_{n}}\left(\Delta_{i_{0} \ldots i_{n}}, \Delta_{j_{0} \ldots j_{n}}\right) \geq \delta$ for any $\left(i_{0} \ldots i_{n}\right) \neq\left(j_{0} \ldots j_{n}\right)$.

The set $Q$ gives the number of basic sets in each step. In the following, we will show that $Q$, together with $\left\{l_{n}\right\}_{n=0}^{\infty}$, provides an estimation of the topological entropy of the limit set (Theorem 4.3). This is due to the intuitive fact that the topological entropy of a set represents the exponential growth of the number of orbit segments that can be distinguished in the Bowen metric $d_{n}$ (with a certain accuracy). First we need a relationship between topological entropy and measure-theoretical entropy. Define the lower local entropy of a probability measure $\mu$ at $x \in X$ as

$$
\underline{h}_{\mu}(f, x)=-\lim _{\epsilon \rightarrow 0} \varlimsup_{n \rightarrow \infty} \frac{1}{n} \log \mu\left(B_{n}(x, \epsilon)\right) .
$$

The upper local entropy $\bar{h}_{\mu}(f, x)$ is defined similarly.

Proposition 4.2. Let $(X, f)$ be a dynamical system, and let $\mu$ be a probability measure on $X$. Then for any Borel subset $Z$ of $X$ with positive measure, we have:

(i) if $\underline{h}_{\mu}(f, x) \geq s$ for $\mu$-almost all $x \in Z$, then $h_{t}(f, Z) \geq s$;

(ii) if $\bar{h}_{\mu}(f, x) \leq s$ for every $x \in Z$, then $h_{t}(f, Z) \leq s$.

We omit the proof as it is analogous to the Hausdorff dimension case by using Definition 2.1 (see [21, Theorem 7.4], [29]). Let diam $\left.\right|_{d_{n}}(E)$ be the diameter of a set $E$ in the Bowen metric $d_{n}$.

THEOREM 4.3. Let $F$ be a Moran fractal defined by $Q=\prod_{k=0}^{\infty}\left\{1, \ldots, c_{k}\right\}$, as in Definition 4.1. Assume that $l_{n}$ in (C3) satisfies $\lim _{n \rightarrow \infty} l_{n+1} / l_{n}=1$. Then for any open set $U$ which has non-empty intersection with $F$, we have

$$
h_{t}(f, F \cap U) \geq \varliminf_{n \rightarrow \infty} \frac{1}{l_{n}}\left(\log \# Q_{n}\right)=\varliminf_{n \rightarrow \infty} \frac{1}{l_{n}} \log \prod_{i=0}^{n} c_{i} .
$$

Moreover, if $f$ is positively expansive, then the equality in (13) holds provided that $r=\varlimsup_{n \rightarrow \infty} \sup _{i_{0} \ldots i_{n}}\left\{\left.\operatorname{diam}\right|_{d_{l n}} \Delta_{i_{0} \ldots i_{n}}\right\}<\gamma / 4$, where $\gamma$ is an expansive constant as in (5).

Proof. For any $i_{0} \ldots i_{n} \in Q_{n}$, define the probability $p\left(i_{0} \ldots i_{n}\right)=\left(\# Q_{n}\right)^{-1}$. By using the Kolmogorov consistency theorem, there exists a probability measure $\mu$ on $F$ such that

$$
\mu\left(\Delta_{i_{0} \ldots i_{n}}\right)=p\left(i_{0} \ldots i_{n}\right)>0 .
$$

Then for any open set $U$ that has non-empty intersection with $F$, we have $\mu(F \cap U)>0$. Suppose $n>l_{0}$ and let $k \geq 0$ be such that $l_{k}<n \leq l_{k+1}$. Observe that for any $x \in F$ and $\epsilon<\delta / 2$ (where $\delta$ is as in (C3)), at most one basic set $\Delta_{i_{0} \ldots i_{k}}$ intersects $B_{n}(x, \epsilon)$. Thus

$$
\mu\left(B_{n}(x, \epsilon)\right)=\mu\left(B_{n}(x, \epsilon) \cap F\right) \leq \mu\left(\Delta_{i_{0} \ldots i_{k}}\right)=\left(\prod_{i=0}^{k} c_{i}\right)^{-1} .
$$


This gives (13) by using (i) of Proposition 4.2.

For the second part of the theorem, we have by Lemma 2.4 that for any $0<\epsilon<r$, there is $N_{0}$ such that

$$
\left.\operatorname{diam}\right|_{d_{l_{k}-N_{0}}} \Delta_{i_{0} \ldots i_{k}}<\epsilon \text { for any } i_{0} \ldots i_{k} \in Q_{k} \text { and } l_{k} \geq N_{0} .
$$

Hence for $x \in F$ and $l_{k}<n \leq l_{k+1}$, there is some $i_{0} \ldots i_{k+1}$ such that $\Delta_{i_{0} \ldots i_{k+1}} \subseteq$ $B_{n-N_{0}}(x, \epsilon)$. Using this and (14), we get the upper bound of $h_{t}(f, F \cap U)$ by applying (ii) of Proposition 4.2.

In the following, we construct a dynamically defined Moran fractal when the system satisfies the SPEC. We will adopt the notations and construction of $\Phi(\mathbf{z})$ in part (iii) near the end of $\S 3$.

Construction $M$. Let $C_{k}=\left\{z_{1}^{k}, \ldots, z_{c_{k}}^{k}\right\}, k \in \mathbb{N}$, be subsets of $X$ with cardinalities $c_{k}$. Let $Q=\prod_{k=0}^{\infty}\left\{1, \ldots, c_{k}\right\}^{N_{k}}$, where $\left\{N_{k}\right\}_{k=0}^{\infty}$ is an arbitrary sequence of numbers in $\mathbb{N}$. For each $\mathbf{i} \in Q$, we define a point $x_{\mathbf{i}}$ as follows. Write $\mathbf{i}$ as

$$
\mathbf{i}=\mathbf{i}_{0} \ldots \mathbf{i}_{k} \ldots \quad \text { with }\left|\mathbf{i}_{k}\right|=N_{k} .
$$

Let

$$
h(\mathbf{i})=\left.\left.\left.\left.z_{\mathbf{i}_{0}(0)}^{0}\right|_{n_{0}} \smile z_{\mathbf{i}_{0}(1)}^{0}\right|_{n_{0}} \cdots \smile z_{\mathbf{i}_{0}\left(N_{0}-1\right)}^{0}\right|_{n_{0}} \bowtie_{0} z_{\mathbf{i}_{1}(0)}^{1}\right|_{n_{1}} \smile \cdots .
$$

be the block sequence as in (12) $\left(\mathbf{i}_{k}(j)\right.$ denotes the $j$ th coordinate of $\left.\mathbf{i}_{k}\right)$, and let $x_{\mathbf{i}}=$ $\Phi(h(\mathbf{i}))$.

For the orbit $\left\{f^{j}\left(x_{\mathbf{i}}\right)\right\}_{j}$, we see from the SPEC and the orbit specification lemma that for the gaps in the $\epsilon$-shadowing corresponding to the part $\smile$, the length of the orbit is $m$, and for the gaps in the $\epsilon_{k}$-shadowing corresponding to $\bowtie_{k}$, the length is $m_{k}$. For convenience of counting, we use the convention of assigning a length $m$ for $\smile$ and $m_{k}$ for $\bowtie_{k}$, as described in the last paragraph of $\S 3$.

Proposition 4.4. Let $F=\left\{x_{i}=\Phi(h(\mathbf{i})): \mathbf{i} \in Q\right\}$. If we assume that the points in $C_{k}$ are $\delta$-separated in the Bowen metric $d_{n_{k}}$, with $\delta>2\left(\epsilon+\epsilon_{0}\right)$ and $\lim _{k \rightarrow \infty}\left(n_{k+1}+\right.$ $\left.m_{k+1}\right) / N_{k}=0$, then $F$ is a Moran fractal modeled by $Q$.

Moreover, if $(X, f)$ is a positively expansive system and $\left\{n_{k}\right\}_{k=0}^{\infty}$ is strictly increasing, then for any open set $U$ that has non-empty intersection with $F$,

$$
\varliminf_{k \rightarrow \infty} \frac{1}{n_{k}} \log c_{k} \leq h_{t}(f, F \cap U) \leq \varlimsup_{k \rightarrow \infty} \frac{1}{n_{k}} \log c_{k} .
$$

Proof. Let $\mathbf{i}=\mathbf{i}_{0} \mathbf{i}_{1} \ldots \in Q$ with $\left|\mathbf{i}_{i}\right|=N_{i}$. For $k \in \mathbb{N}$, we let

$$
y_{\mathbf{i}_{k}}=\Phi_{\epsilon}\left(\left.z_{\mathbf{i}_{k}(0)}^{k}\right|_{n_{k}}, \ldots,\left.z_{\mathbf{i}_{k}\left(N_{k}-1\right)}^{k}\right|_{n_{k}}\right)
$$

and then let

$$
x_{\mathbf{i}_{0} \cdots \mathbf{i}_{k}}=\Phi_{\left\{\epsilon_{k}\right\}}\left(y_{\mathbf{i}_{0}}, y_{\mathbf{i}_{1}}, \ldots, y_{\mathbf{i}_{k}}\right) .
$$

Define $t_{k}=\sum_{i=0}^{k} N_{i}-1$; this is the index such that $\mathbf{i}_{0} \ldots \mathbf{i}_{k}=i_{0} \ldots i_{t_{k}}$. Let $l_{n}$ be the length of the sequence in (15) up to the $n$th block; then

$$
l_{n}= \begin{cases}\sum_{i=0}^{k}\left(n_{i}^{\prime}+m_{i}\right)-m_{k} & \text { if } n=t_{k} \text { for some } k, \\ \sum_{i=0}^{k}\left(n_{i}^{\prime}+m_{i}\right)+\left(n-t_{k}\right)\left(m+n_{k+1}\right)-m & \text { if } t_{k}<n<t_{k+1},\end{cases}
$$


where $n_{i}^{\prime}=N_{i}\left(n_{i}+m\right)-m$. For any $i_{0} \ldots i_{n} \in Q_{n}$, let

$$
\Delta_{i_{0} \ldots i_{n}}= \begin{cases}\overline{B_{t_{t_{k}}}\left(x_{i_{0} \ldots i_{t_{k}}}, 2 \epsilon_{k}\right)} & \text { if } n=t_{k}, \\ \bigcup_{i_{n+1}, \ldots, i_{t_{k}}} \Delta_{i_{0} \cdots i_{t_{k}}} & \text { if } t_{k-1}<n<t_{k} .\end{cases}
$$

By using Lemma 3.2 and Corollary 3.3, we can easily check that $F$ and $\left\{\Delta_{i_{0} \ldots i_{n}}\right\}$ satisfy the requirement of Definition 4.1. The last statement in the proposition follows from Theorem 4.3 , by observing that $\log \# Q_{n}=\sum_{i=0}^{k} N_{i} \log c_{i}+\left(n-t_{k}\right) \log c_{k+1}$ and

$$
\lim _{n \rightarrow \infty} \frac{1}{l_{n}}\left(\sum_{i=0}^{k} N_{i} n_{i}+\left(n-t_{k}\right) n_{k+1}\right)=1,
$$

where $k$ is such that $t_{k} \leq n<t_{k+1}$.

As an application of the theorem, we show that for dynamical systems which are positively expansive and which satisfy the SPEC, there are plenty of Moran fractals by Construction M with full topological entropy. Moreover, we have good control of the $m_{k}$ 's, i.e. the lengths of the $\bowtie_{k}$ gaps. We need the following lemma.

LEMMA 4.5. Let $(X, f)$ be a positively expansive system (with d as in (6)) which satisfies the specification condition. For $0<\epsilon<\eta, p>1$ and $k \in \mathbb{N}$,

$$
m\left(\frac{\epsilon}{k^{p}}\right) \leq p \frac{\log k}{\log \lambda}+2+m(\epsilon) .
$$

Proof. Let $N=[p \log k / \log \lambda]+2$ (where $[a]$ is the integer part of $a$ ). By invoking the expansive property of $d$ in (6) repeatedly, we deduce from $d_{N}(x, y)<\epsilon$ that

$$
\lambda^{N-1} d(x, y) \leq d\left(f^{N-1}(x), f^{N-1}(y)\right)<d_{N}(x, y)<\epsilon,
$$

i.e. $d(x, y)<k^{-p} \epsilon$. For any $I_{j}=\left[a_{j}, a_{j}+n_{j}\right](j \leq n)$ with $\operatorname{dist}\left(I_{i}, I_{j}\right)>m(\epsilon)+N$, we let $I_{j}^{\prime}=\left[a_{j}, a_{j}+n_{j}+N\right](j \leq n)$. Then any point that $\epsilon$-shadows $y_{j}$ in $I_{j}^{\prime}$ must $k^{-p} \epsilon$ shadow $y_{j}$ in $I_{j}$. The conclusion follows from the definition of $m(\cdot)$.

Proposition 4.6. Let $(X, f)$ be a positively expansive system which satisfies the specification condition. There exists a subset $F \subseteq X$ by Construction $M$, with $m_{k} \leq O(k)$, such that $h_{t}(f, F)=h_{t}(f)$.

Proof. We may assume the metric $d$ on $X$ satisfies (6). Take $\epsilon<\frac{1}{5} \min \{\eta, \gamma / 4\}$, where $\eta$ is from (6) and $\gamma$ is an expansive constant as in (5). Let $p>1$. By Lemma 4.5 , there is $K$ such that

$$
m\left(\frac{\epsilon}{k^{p}}\right) \leq k+m(\epsilon) \quad \text { for } k \geq K
$$

Let $\epsilon_{k}=\sum_{j=k+K}^{\infty} \epsilon / j^{p}$. As $k$ increases, $\epsilon_{k}$ decreases to zero since $\sum_{k=1}^{\infty} \epsilon / k^{p}<\infty$. By taking $K$ to be large, we can assume $\epsilon_{0}<\frac{1}{4} \epsilon$. Moreover, $m_{k}=m\left(\epsilon_{k}-\epsilon_{k+1}\right)$ satisfies

$$
m_{k}=m\left(\frac{\epsilon}{(k+K)^{p}}\right) \leq k_{0}+k \quad \text { where } k_{0}=K+m(\epsilon) .
$$


Let $\left\{n_{k}\right\}_{k=0}^{\infty}$ and $\left\{N_{k}\right\}_{k=0}^{\infty}$ be strictly increasing sequences with $\lim _{k \rightarrow \infty}\left(n_{k+1} / N_{k}\right)=0$. We take $C_{k}$ to be any maximal $\left(n_{k}, \delta\right)$-separated set with $2\left(\epsilon+\epsilon_{0}\right)<\delta<\gamma / 4$. By Theorem 2.5,

$$
\lim _{k \rightarrow \infty} \frac{1}{n_{k}} \log \# C_{k}=h_{t}(f) .
$$

Hence the set $F$ defined by Construction M using $\epsilon,\left\{\epsilon_{k}\right\},\left\{n_{k}\right\},\left\{N_{k}\right\}$ and $C_{k}$ above has entropy $h_{t}(f)$ by Proposition 4.4.

\section{The proof of the main theorem}

In this section we give a proof of our main result, Theorem 1.1. The technique is to construct a Moran fractal, arbitrarily close to having full entropy, such that each of its elements has the pre-assigned upper and lower exponential recurrence rates. Such a fractal is derived from the one in Proposition 4.6. The construction for obtaining the right rates is rather complicated notation-wise. We will therefore present it in three subsections, which deal with three cases of increasing complexity: the full symbolic space, the general symbolic space, and finally the dynamical system $(X, f)$ with the SPEC. In order to make the main idea of the proof clearer, we leave some of the not-so-essential and tedious lemmas to Appendix A.

5.1. The full symbolic space. Let $\left\{p_{k}\right\}_{k=0}^{\infty}$ and $\left\{q_{k}\right\}_{k=0}^{\infty}$ be two strictly increasing sequences with $p_{k+1}<q_{k}$. We define two other sequences $\left\{p_{k}^{\prime}\right\}_{k=0}^{\infty}$ and $\left\{q_{k}^{\prime}\right\}_{k=0}^{\infty}$ as follows: set $p_{-1}^{\prime}=1, q_{-1}=0$, and let

$$
p_{k}^{\prime}=p_{k}+\sum_{q_{i}<p_{k}}\left(p_{i}^{\prime}+1\right), \quad q_{k}^{\prime}=q_{k}+\sum_{i<k}\left(p_{i}^{\prime}+1\right) .
$$

It is easy to see that $p_{i}<q_{j}$ if and only if $p_{i}^{\prime}<q_{j}^{\prime}$.

LEMMA 5.1. For any $0 \leq \alpha \leq \beta \leq \infty$, there exist $\left\{p_{k}\right\}_{k=0}^{\infty}$ and $\left\{q_{k}\right\}_{k=0}^{\infty}$ with $p_{k+1}<q_{k}$ such that the sequences $\left\{p_{k}^{\prime}\right\}_{k=0}^{\infty},\left\{q_{k}^{\prime}\right\}_{k=0}^{\infty}$ defined by (16) satisfy (for each $k$ )

$$
\begin{gathered}
p_{k}^{\prime}<q_{k}-q_{k-1}, \\
\lim _{k \rightarrow \infty} \sum_{i=-1}^{k} p_{i}^{\prime} / q_{k}=0, \\
\lim _{k \rightarrow \infty} p_{k}^{\prime} / p_{k-1}^{\prime}=1, \\
\underline{\lim }\left(\log q_{k}^{\prime}\right) / p_{k}^{\prime}=\alpha, \quad \varlimsup_{k \rightarrow \infty}\left(\log q_{k}^{\prime}\right) / p_{k}^{\prime}=\beta .
\end{gathered}
$$

This elementary proof is given in Appendix A. (We can take, for example, $p_{k}=k$ and $q_{k}=C\left[e^{\alpha p_{k}}\right]$ for some $C>1$ when $0<\alpha=\beta<\infty$.) Now let $\Sigma^{\mathbb{N}}$ be an infinite product of finite symbols with the shift map $S$. It is well known that $\left(\Sigma^{\mathbb{N}}, S\right)$ is a positively expanding dynamical system and that the canonical cylinders coincide with the Bowen balls. Moreover, $\left(\Sigma^{\mathbb{N}}, S\right)$ has the SPEC, and the words can be joined together directly. We want to construct a set of $\mathbf{y} \in \Sigma^{\mathbb{N}}$ so that the recurrence rates satisfy

$$
\underline{\tau}(\mathbf{y})=\alpha \quad \text { and } \quad \bar{\tau}(\mathbf{y})=\beta .
$$




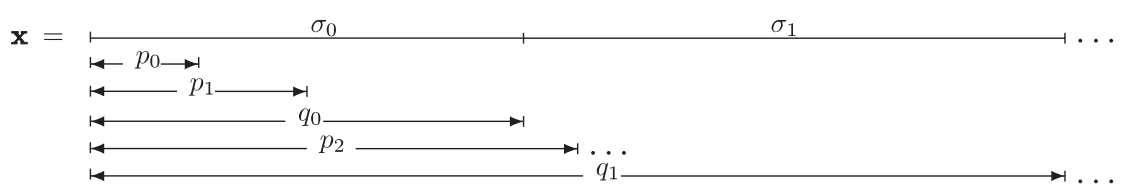

FIGURE 2. The positions of the $p_{k}$ and $q_{k}$.

The idea is similar to that in [15], and involves adding in the initial pieces of the given sequence $\mathbf{y}$ recursively. In our construction, however, the places to insert such pieces are fixed beforehand; this is needed in the general case.

We proceed inductively with the construction . Let $\mathcal{A}$ be a proper subset of $\Sigma$. Let $\left\{p_{k}\right\}_{k=0}^{\infty}$ and $\left\{q_{k}\right\}_{k=0}^{\infty}$ be as in Lemma 5.1. Choose $\mathbf{x}=\mathbf{x}(0) \mathbf{x}(1) \ldots \in \mathcal{A}^{\mathbb{N}}$ (where $\mathbf{x}(i)$ denotes the $i$ th coordinate of $\mathbf{x}$ ) and write it as

$$
\mathbf{x}=\sigma_{0} \sigma_{1} \ldots \sigma_{k} \ldots \quad \text { with }\left|\sigma_{0} \ldots \sigma_{k}\right|=q_{k}
$$

(see Figure 2) and also as

$$
\mathbf{x}=\tau_{0} \tau_{1} \ldots \tau_{k} \ldots \quad \text { with }\left|\tau_{0} \ldots \tau_{k}\right|=p_{k} .
$$

Our construction is to recursively insert the $\tau_{0} \ldots \tau_{k}$ of $\mathbf{x}$ between the $\sigma_{k}$ and $\sigma_{k+1}$ as follows. Let $\left\{p_{k}^{\prime}\right\}_{k=0}^{\infty}$ and $\left\{q_{k}^{\prime}\right\}_{k=0}^{\infty}$ be defined as in (16). We choose $a \in \mathcal{A}, b \in \mathcal{A}^{c}$ and $w_{0}=b b$ as 'markers' for the recurrence. Let $\mathbf{x}_{0}=w_{0} \sigma_{0}$. Suppose we have constructed $w_{i}$ and $\mathbf{x}_{i}$ with $\left|w_{i}\right|=p_{i-1}^{\prime}+1$ and $\left|\mathbf{x}_{i}\right|=q_{i}^{\prime}$ for $i \leq k$. Inductively, let $\mathbf{x}_{k+1}=\mathbf{x}_{k} w_{k+1} \sigma_{k+1}$, where

$$
w_{k+1}= \begin{cases}\left.\left(\mathbf{x}_{k}\right)\right|_{p_{k}^{\prime}} a & \text { if } p_{k} \leq q_{i}<p_{k+1} \text { for some } i, \\ \left.\left(\mathbf{x}_{k}\right)\right|_{p_{k}^{\prime}} b & \text { otherwise. }\end{cases}
$$

Hence $\left|w_{k+1}\right|=p_{k}^{\prime}+1$ and $\left|\mathbf{x}_{k+1}\right|=q_{k+1}^{\prime}$. It follows that $p_{k}^{\prime}$ and $q_{k}^{\prime}$ satisfy the equations in (16).

Finally, we let

$$
\Lambda(\mathbf{x})=\bigcap_{k=0}^{\infty}\left[\mathbf{x}_{k}\right]=w_{0} \sigma_{0} w_{1} \ldots \sigma_{k} w_{k+1} \ldots
$$

and let $\Lambda\left(\mathcal{A}^{\mathbb{N}}\right)$ denote all the $\Lambda(x)$, with $x \in \mathcal{A}^{\mathbb{N}}$. We define the first return time for the shift map $S$ on $\Sigma^{\mathbb{N}}$ with respect to $\left.\mathbf{y}\right|_{p_{k}^{\prime}}$ as

$$
R_{k}(\mathbf{y})=\min \left\{i>0:\left.S^{i}(\mathbf{y})\right|_{p_{k}^{\prime}}=\left.\mathbf{y}\right|_{p_{k}^{\prime}}\right\} \quad \text { for all } \mathbf{y} \in \Sigma^{+}, k \geq 0 .
$$

As an illustration, we shall use the $\mathbf{x}=\sigma_{0} \sigma_{2} \ldots=\tau_{0} \tau_{1} \ldots$ in Figure 2 to explain the construction and the first return time. According to the construction,

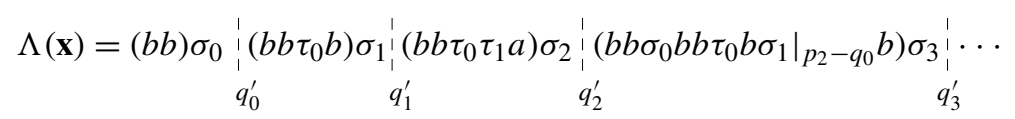

(the symbols within parentheses are the inserted segments). Letting $\mathbf{y}=\Lambda(\mathbf{x})$, we have

$$
\begin{gathered}
\left.\mathbf{y}\right|_{p_{0}^{\prime}}=(b b) \tau_{0}=\left.(b b) \sigma_{0}\right|_{p_{0}}, \\
\left.\mathbf{y}\right|_{p_{1}^{\prime}}=(b b) \tau_{0} \tau_{1}=\left.(b b) \sigma_{0}\right|_{p_{1}}, \\
\left.\mathbf{y}\right|_{p_{2}^{\prime}}=\left.(b b) \tau_{0} \tau_{1} \tau_{2}\right|_{q_{0}-p_{1}}\left(b b \tau_{0} b\right) S^{q_{0}-p_{1}}\left(\tau_{2}\right)=\left.(b b) \sigma_{0}\left(b b \tau_{0} b\right) \sigma_{1}\right|_{p_{2}-q_{0}} .
\end{gathered}
$$

\section{CAmbridge J J JPNALS}


(Note that from Figure 2, $\left.\sigma_{0} \sigma_{1}\right|_{p_{2}-q_{0}}=\tau_{0} \tau_{1} \tau_{2}$.) Write $R_{i}=R_{i}(\mathbf{y})$. It is clear that $R_{0} \leq R_{1} \leq R_{2}$ and $R_{i} \leq q_{i}^{\prime}$ for $i=0,1$, 2. Since $b$ does not appear in $\sigma_{0}$, we have $R_{0}=q_{0}^{\prime}$. Next, observe that $R_{1} \neq R_{0}$ since $\tau_{1}$ does not contain $b$ and hence $\left(b b \tau_{0} b\right)$ is not a prefix of $\left.\mathbf{y}\right|_{p_{1}^{\prime}}$. Then we must have $R_{1}=q_{1}^{\prime}$, because otherwise $\left.\mathbf{y}\right|_{2}=b b$ would appear in $b \tau_{0} b \sigma_{1}$ or $b$ would be the end symbol of $\sigma_{1}$, contradicting the fact that $\tau_{0}, \sigma_{1}$ do not contain $b$. We have $R_{2} \neq R_{1}$, since the subword $\left(b b \tau_{0} b\right)$ of $\left.\mathbf{y}\right|_{p_{2}^{\prime}}$ does not appear in $\sigma_{2}$. Notice that $\tau_{0} \tau_{1} a \sigma_{2}$ is composed of symbols from $\mathcal{A}$; this forces $R_{2}=q_{2}^{\prime}$, since otherwise $\left.\mathbf{y}\right|_{2}=b b$ would appear in $b \tau_{0} \tau_{1} a \sigma_{2}$ or $b$ would be the suffix of $\sigma_{2}$.

Proposition 5.2. $R_{k}(\mathbf{y})=q_{k}^{\prime}$ for $\mathbf{y} \in \Lambda\left(\mathcal{A}^{\mathbb{N}}\right)$. Consequently,

$$
\underline{\tau}(\mathbf{y})=\alpha \quad \text { and } \quad \bar{\tau}(\mathbf{y})=\beta \quad \text { for all } \mathbf{y} \in \Lambda\left(\mathcal{A}^{\mathbb{N}}\right) .
$$

Proof. We express $\mathbf{y}$ as

$$
\mathbf{y}=\underset{\left|\longleftarrow----q_{k}^{\prime}-----\rightarrow\right|}{w_{0} \sigma_{0} w_{1} \sigma_{1} w_{2} \ldots \sigma_{k} w_{k+1} \ldots}
$$

Note that $\left|w_{k+1}\right|=p_{k}^{\prime}+1$. It is clear that if $n=q_{k}^{\prime}$, then

$$
\left.S^{n}(\mathbf{y})\right|_{p_{k}^{\prime}}=\left.w_{k+1}\right|_{p_{k}^{\prime}}=\left.y\right|_{p_{k}^{\prime}} .
$$

The proof of $q_{k}^{\prime}$ being smallest follows from a direct check of the construction and an inductive argument; it is omitted here, but the details can be found in [29] (the idea is the same as in the above illustration). We obtain (22) by applying Corollary 2.7 to $\mathbf{y}$ and using $R_{k}(\mathbf{y})=q_{k}^{\prime}$ together with Lemma 5.1.

Proof of Theorem 1.1 in the case of the full symbolic space. The result in this case was already known in [15]. We provide this new proof in order to motivate the more complicated case of a dynamical system in $\$ 5.3$.

Assume $0,1 \in \Sigma$. Let $n \in \mathbb{N}$ be fixed and let $\mathcal{A}=\left\{0 a_{0} a_{1} \ldots a_{n-1} 0: a_{j} \in \Sigma\right\}$. The set $\mathcal{A}$ consists of words of length $n+2$ which begin and end with 0 . (Here $\mathcal{A} \subset \Sigma^{n+2}$ instead of $\Sigma$, but this does not affect the construction of $\Lambda$ and the recurrence behavior.) Consider $\mathcal{A}^{\mathbb{N}}$; this is a dynamically defined Moran fractal with $Q=\prod_{0}^{\infty}\{1,2, \ldots, \# \mathcal{A}\}$ and $l_{k}=(n+2) k$, and the $k$ th level sets are cylinders $[\tau]$ with $\tau \in \mathcal{A}^{k}$. Theorem 4.3 then yields

$$
h_{t}\left(S, \mathcal{A}^{\mathbb{N}}\right)=\frac{1}{n+2} \log \# \mathcal{A}=\frac{n}{n+2} h_{t}(S),
$$

where $h_{t}(S)=\log \# \Sigma$. Hence for any $\epsilon>0, h_{t}\left(S, \mathcal{A}^{\mathbb{N}}\right)>h_{t}(S)-\epsilon$ for large $n$.

Next, we let

$$
a=00 \ldots 0 \in \mathcal{A} \text { and } b=11 \ldots 1 \in \mathcal{A}^{c}
$$

be words made up of 0 or 1 , with length $n+2$. We change the definition of $\left\{p_{k}^{\prime}\right\}$ and $\left\{q_{k}^{\prime}\right\}$ accordingly: set $p_{-1}^{\prime}=|b|=n+2, q_{-1}=0$ and let

$$
p_{k}^{\prime}=p_{k}+\sum_{q_{i}<p_{k}}\left(p_{i}^{\prime}+|b|\right), \quad q_{k}^{\prime}=q_{k}+\sum_{i<k}\left(p_{i}^{\prime}+|b|\right) .
$$

Given $0 \leq \alpha \leq \beta \leq \infty$, let $\left\{p_{k}\right\}$ and $\left\{q_{k}\right\}$ satisfy Lemma 5.1. We have by Proposition 5.2 that $\Lambda\left(\mathcal{A}^{\mathbb{N}}\right) \subseteq D(\alpha, \beta)$ and hence $h_{t}(S, D(\alpha, \beta)) \geq h_{t}\left(S, \Lambda\left(\mathcal{A}^{\mathbb{N}}\right)\right)$. 


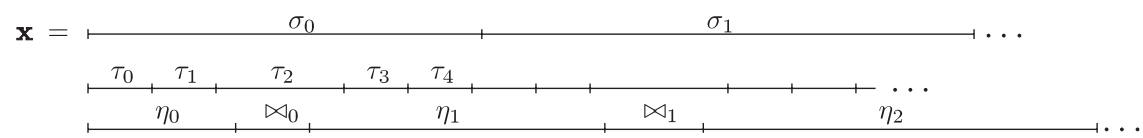

FIGURE 3. The positions of $\sigma_{k}, \tau_{k}, \eta_{k}$ and $\bowtie_{k}$.

Finally, we estimate the entropy of $\Lambda\left(\mathcal{A}^{\mathbb{N}}\right)$. We see that $\Lambda\left(\mathcal{A}^{\mathbb{N}}\right)$ is also a dynamically defined Moran fractal with $Q^{\prime}=Q$ and $l_{k}^{\prime}=l_{k}+\Sigma_{q_{i}<l_{k}} p_{i}^{\prime}$, and the $k$ th level sets are cylinders of length $l_{k}^{\prime}$ that intersect $\Lambda\left(\mathcal{A}^{\mathbb{N}}\right)$. Hence

$$
h_{t}\left(S, \Lambda\left(\mathcal{A}^{\mathbb{N}}\right)\right) \geq \varliminf_{k \rightarrow \infty} \frac{1}{l_{k}^{\prime}} \log \# Q_{n}^{\prime} \geq \lim _{k \rightarrow \infty} \frac{l_{k}}{l_{k}^{\prime}} h_{t}\left(S, \mathcal{A}^{\mathbb{N}}\right) .
$$

Since $\lim _{k \rightarrow \infty} \sum_{i \leq k} p_{i}^{\prime} / q_{k}=0$, it follows that $\underline{\lim }_{k \rightarrow \infty} l_{k} / l_{k}^{\prime}=1$ and

$$
h_{t}(S, D(\alpha, \beta)) \geq h_{t}\left(S, \Lambda\left(\mathcal{A}^{\mathbb{N}}\right)\right)>h_{t}(S)-\epsilon .
$$

As $\epsilon>0$ is arbitrary, this shows $h_{t}(S, D(\alpha, \beta))=h_{t}(S)$ and the theorem is proved.

5.2. The general symbolic space. We generalize the construction in $§ 5.1$ to some Moran fractals in the symbolic space; the procedure is similar to that in Construction M. This construction will be extended to the more elaborate case of dynamical systems in $§ 5.3$. Let $\mathcal{A}$ be a proper subset of $\Sigma$ and let $\mathcal{A}_{*}$ denote the set of finite words admissible in $\mathcal{A}^{\mathbb{N}}$. We will consider those $\mathbf{x}$ of $\Sigma^{\mathbb{N}}$ that can be expressed as

$$
\mathbf{x}=\underset{1 \leftarrow--\bowtie_{0}}{ } \eta_{1} \bowtie_{1} \ldots \eta_{k-1} \bowtie_{k-1} \ldots,
$$

where $\eta_{k}$ belongs to $\mathcal{A}_{*}$ with $\left|\eta_{k}\right|=n_{k}^{\prime}$ and $\left|\eta_{0} \bowtie_{0} \ldots \eta_{k-1} \bowtie_{k-1}\right|=\ell_{k}$. We also let $\left|\bowtie_{k}\right|=m_{k}$. (Note that $\bowtie_{k}$ may not belong to $\mathcal{A}_{*}$. The expression above is an extension of the one in $\$ 5.1$ where $\bowtie_{k}$ 's do not appear; moreover, the expression resembles the sequence in (15) without the $\smile$ 's.) It is clear that $n_{k}^{\prime}+\ell_{k}<\ell_{k+1}$.

As in the previous section, we write $\mathbf{x} \in \Sigma^{\mathbb{N}}$ as

$$
\begin{aligned}
\mathbf{x} & =\sigma_{0} \sigma_{1} \ldots \sigma_{k} \ldots \quad \text { with }\left|\sigma_{0} \ldots \sigma_{k}\right|=q_{k}, \\
\text { and } \quad & \mathbf{x}=\tau_{0} \tau_{1} \ldots \tau_{k} \ldots \quad \text { with }\left|\tau_{0} \ldots \tau_{k}\right|=p_{k} .
\end{aligned}
$$

The specific choice of the above sequences comes from Lemma 5.3. For example, we can take $n_{k}^{\prime}=O\left(\left[e^{k^{4}}\right]\right)$ and $m_{k}=k$ so that $n_{k}^{\prime}$ dominates the length of $\ell_{k+1}$; let $\left\{p_{k}\right\}$ be the set of numbers (of an arithmetic progression) satisfying (29), and choose $q_{k}$ to satisfy Lemma 5.3 (e.g. $q_{k} \approx O\left(\left[e^{\alpha p_{k}}\right]\right.$ ) in the case where $\left.0<\alpha=\beta<\infty\right)$. Comparing with (23) (see Figure 3), we see that it could happen that $\sigma_{k}, \tau_{k} \notin \mathcal{A}_{*}$; hence Proposition 5.2 does not follow directly. Thus we need to modify the previous construction slightly to accommodate the extra $\bowtie_{k}$ 's.

Take $a \in \mathcal{A}, b \in \mathcal{A}^{c}$ and $w_{0}=b b$ as before. Assume $\sigma_{0}$ does not contain $w_{0}$ as a segment (otherwise adjust $w_{0}=b b \ldots b$ to be longer). We use induction to construct sequences $\left\{\mathbf{x}_{k}\right\},\left\{w_{k}\right\},\left\{p_{k}^{\prime}\right\},\left\{q_{k}^{\prime}\right\},\left\{\sigma_{k}^{\prime}\right\}$ and $\left\{r_{j}\right\}$ for $l_{j} \leq q_{k}$ (which is used as 


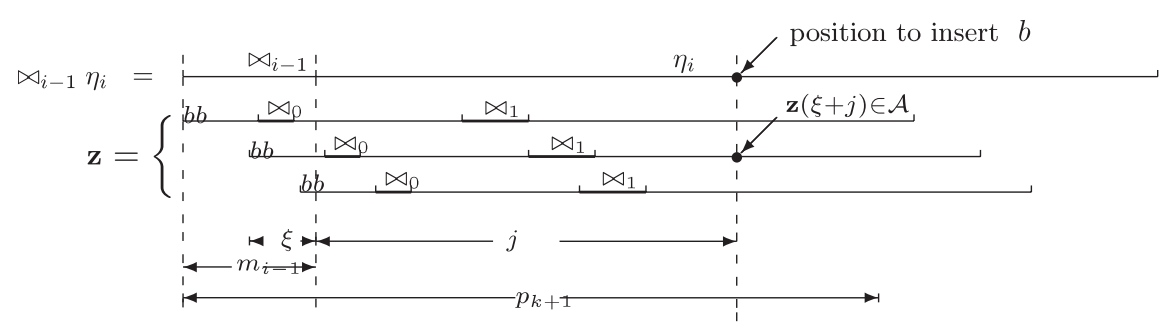

FIGURE 4. The positions in $\mathbf{z}$ and the insertion of $b$.

an indicator function). We take $\ell_{0}<q_{0}$. Let $p_{-1}^{\prime}=1$ and $q_{-1}=0$, and set $\sigma_{0}^{\prime}=\sigma_{0}$, $\mathbf{x}_{0}=w_{0} \sigma_{0}$ and $r_{i}=\infty$ for $\ell_{i} \leq q_{0}$. Suppose we have already constructed the sequences up to the $k$ th step, such that

$$
\begin{aligned}
& p_{k}^{\prime}=p_{k}+\sum_{q_{j}<p_{k}}\left(p_{j}^{\prime}+1\right)+\sum_{r_{j}<p_{k}} 1, \\
& q_{k}^{\prime}=q_{k}+\sum_{q_{j}<q_{k}}\left(p_{j}^{\prime}+1\right)+\sum_{r_{j}<q_{k}} 1 .
\end{aligned}
$$

We proceed to the $(k+1)$ th step as follows. Let

$$
\sigma_{k+1}=\eta_{s}^{T} \bowtie_{s} \eta_{s+1} \ldots \bowtie_{t-1} \eta_{t}^{T},
$$

where $\eta_{s}^{T}$ and $\eta_{t}^{T}$ are truncated segments from $\eta_{s}$ and $\eta_{t}$, respectively.

(i) We will modify the above $\eta_{i}$ in $\sigma_{k+1}$ to $\eta_{i}^{\prime}$ so that the new piece $\bowtie_{i} \eta_{i}^{\prime}$ does not contain $\mathbf{z}=\left.\mathbf{x}_{k}\right|_{p_{k+1}^{\prime}}$ as a segment. (Note that $p_{k+1}^{\prime}$ is defined since $p_{k+1}<q_{k}$ and $r_{i}$ with $l_{i} \leq q_{k}$ has been determined in the $k$ th step.)

Observe that $\left.\mathbf{z}\right|_{2}=b b$ does not appear in the $\eta_{i}$; hence the only situation in which $\sigma_{k+1}$ may contain $\mathbf{z}$ is as shown in Figure 4 for some $l \leq m_{i-1}$. Thus, we consider $\eta_{i}$ with $s+1 \leq i \leq t$. If $b$ appears in $S^{m_{i-1}}(\mathbf{z})$ (not counting $b$ in the $\triangleleft_{k}$ 's in $\mathbf{z}$ ), we let $\eta_{i}^{\prime}=\eta_{i}$ and $r_{i}=\infty$; otherwise, we insert $b$ into $\eta_{i}$ at position $j$ to form $\eta_{i}^{\prime}$, i.e.

$$
\eta_{i}^{\prime}=\eta_{i}(0) \ldots \eta_{i}(j-1) b \eta_{i}(j) \ldots \eta_{i}\left(n_{i}^{\prime}-1\right)
$$

where $j \in\left(m_{i-1}, p_{k+1}\right)$ is such that

$$
\mathbf{z}(\xi+j) \in \mathcal{A} \text { for any } 1 \leq \xi \leq m_{i-1} .
$$

Let $r_{i}=\ell_{i}+j$. The existence of such $j$ follows from the choice of the sequences $\left\{p_{i}\right\}$, $\left\{q_{i}\right\},\left\{n_{i}^{\prime}\right\}$ and $\left\{\ell_{i}\right\}$ (Lemma A.2 in Appendix A). Intuitively, the symbols of $S^{m_{i-1}}(\mathbf{z})$ belong to $\mathcal{A}$ except for those in the $\bowtie_{i}$. Since the total length of such $\bowtie_{i}$ 's is very small compared with that of $S^{m_{i-1}}(\mathbf{z})$, there must exist some $j$ such that (28) holds.

(ii) We define $\sigma_{k+1}^{\prime}=\sigma_{k+1}$ and $r_{s}=\infty$ if $\sigma_{k+1}=\eta_{s}^{T}$ in (26); otherwise, let

$$
\sigma_{k+1}^{\prime}=\eta_{s}^{T} \bowtie_{s} \eta_{s+1}^{\prime} \cdots \bowtie_{t-1}{\eta_{t}^{\prime}}^{T},
$$

using (i). Note that no segment of $\sigma_{k+1}^{\prime}$ equals $\left.\mathbf{x}_{k}\right|_{p_{k+1}^{\prime}}$. We then define

$$
\mathbf{x}_{k+1}=\mathbf{x}_{k} w_{k+1} \sigma_{k+1}^{\prime},
$$


where

$$
w_{k+1}= \begin{cases}\left.\left(\mathbf{x}_{k}\right)\right|_{p_{k}^{\prime}} a & \text { if } p_{k} \leq q_{i}<p_{k+1} \text { for some } i \\ \left.\left(\mathbf{x}_{k}\right)\right|_{p_{k}^{\prime}} b & \text { otherwise }\end{cases}
$$

is as in (21).

Finally, we define, for $\mathbf{x}$ in (23),

$$
\Lambda^{\prime}(\mathbf{x})=\bigcap_{k=0}^{\infty}\left[\mathbf{x}_{k}\right]=w_{0} \sigma_{0}^{\prime} w_{1} \ldots \sigma_{k}^{\prime} w_{k+1} \ldots
$$

As in $\$ 5.1$, we need to choose $\left\{p_{k}\right\}$ and $\left\{q_{k}\right\}$ to have the desired properties.

LemmA 5.3. For any $0 \leq \alpha \leq \beta \leq \infty$, there exist $\left\{p_{k}\right\}_{k=0}^{\infty}$ and $\left\{q_{k}\right\}_{k=0}^{\infty}$ with $p_{k+1}<q_{k}$,

$$
\begin{gathered}
\ell_{i}<p_{k}<\ell_{i}+n_{i}^{\prime} \text { for some } i \\
\text { and } \ell_{j}+4 p_{k}<q_{k}<\ell_{j}+n_{j}^{\prime}-4 p_{k} \text { for some } j ;
\end{gathered}
$$

the sequences $\left\{p_{k}^{\prime}\right\}_{k=0}^{\infty},\left\{q_{k}^{\prime}\right\}_{k=0}^{\infty}$ defined by (24) and (25) satisfy $p_{k}^{\prime}<3 p_{k}$ and (18)-(20).

The proof is in Appendix A, modified from Lemma 5.1.

Note that $p_{k}^{\prime}<3 p_{k}$, and (30) implies (17).

Proposition 5.4. Let $F$ be the set of $\mathbf{x}$ in (23). Then, for any $\mathbf{y} \in F^{\prime}=\Lambda^{\prime}(F)$, we have $R_{k}(\mathbf{y})=q_{k}^{\prime}$ and

$$
\underline{\tau}(\mathbf{y})=\alpha, \quad \bar{\tau}(\mathbf{y})=\beta .
$$

The proof of this proposition is again deferred to Appendix A. We note that, by using Theorem 4.3, it can be shown that the set $F$ is a Moran fractal with the same entropy as $\mathcal{A}^{\mathbb{N}}$ (which is $\log \# \mathcal{A}$ ) if $\lim _{n \rightarrow \infty} \sum_{l_{i}<n} m_{i} / n=0$; the same is true for $F^{\prime}$ if, moreover, $\lim _{k \rightarrow \infty} \sum_{i \leq k} p_{i}^{\prime} / q_{k}=0$.

5.3. The dynamical system case. Let $(X, f)$ be a dynamical system with the SPEC, and let $F$ be the Moran fractal as in Proposition 4.6, consisting of $x_{\mathbf{i}}=\Phi(h(\mathbf{i}))$ with

$$
h(\mathbf{i})=\eta_{0}^{0} \bowtie_{0} \eta_{1}^{0} \bowtie_{1} \ldots \bowtie_{k-1} \eta_{k}^{0} \ldots
$$

and $\eta_{k}^{0}=\left.\left.\left.z_{k 1}\right|_{n_{k}} \smile z_{k 2}\right|_{n_{k}} \cdots \smile z_{k N_{k}}\right|_{n_{k}}$. We shall convert each $x_{\mathbf{i}}$ into another $x_{\mathbf{i}}^{\prime}$ which satisfies

$$
\underline{\tau}\left(x_{\mathbf{i}}^{\prime}\right)=\alpha, \quad \bar{\tau}\left(x_{\mathbf{i}}^{\prime}\right)=\beta .
$$

This is done by adapting the main idea in the previous section to change $h(\mathbf{i}) \in \mathcal{X}^{\mathbb{N}}$ into another sequence in $G(h(\mathbf{i})) \in \mathcal{X}^{\mathbb{N}}$ that has the specified recurrence rate; the SPEC (i.e., $\Phi$ ) is then used to push the sequence down into $X$ and to obtain $F^{\prime}$ (see item (iii) near the end of $\S 3$ ).

We let $\epsilon>0$ and $\epsilon_{0}<\epsilon / 4$. For fixed $n \in \mathbb{N}$, let $\left\{n_{k}\right\},\left\{N_{k}\right\}$ be the two sequences in the Moran set $F$ as given in Proposition 4.6; for example, we can take

$$
n_{k}=n\left(k+k_{0}\right)^{4} \quad \text { and } \quad N_{k}=\left[e^{\left(k+k_{0}\right)^{4}}\right] \text {, }
$$

with $m_{k} \leq k+k_{0}$. Let $u^{*}, v^{*} \in X$ be such that $d\left(u^{*}, \overline{O_{f}\left(v^{*}\right)}\right)>8 \epsilon(\epsilon \ll \gamma / 4$, where $\gamma$ is the expansive constant in (5)). 
The construction of $G(h(\mathbf{i}))$ is as follows. Recall that the symbol $\smile$ represents the $\epsilon$-shadow and is assigned a length $m=m(\epsilon)$. First we change $h(\mathbf{i})$ slightly. For each $\eta_{k}^{0}$, define $\eta_{k}$ by breaking each $\left.z_{k j}\right|_{n_{k}}$ into segments of orbits of equal length $n$ and then inserting $\smile u^{*} \smile$ in between, so that

$$
\begin{aligned}
\eta_{k}= & \left.\left.\left.z_{k 1}\right|_{n} \smile u^{*} \smile f^{n}\left(z_{k 1}\right)\right|_{n} \smile u^{*} \smile \cdots \smile u^{*} \smile f^{n_{k}-n}\left(z_{k 1}\right)\right|_{n} \smile u^{*} \\
& \left.\left.\left.\smile \cdots z_{k N_{k}}\right|_{n} \smile u^{*} \smile f^{n}\left(z_{k N_{k}}\right)\right|_{n} \smile u^{*} \smile \cdots \smile u^{*} \smile f^{n_{k}-n}\left(z_{k N_{k}}\right)\right|_{n} .
\end{aligned}
$$

In this way, $\smile u^{*} \smile$ appears periodically in $\eta_{k}$. Let

$$
G_{1}(h(\mathbf{i}))=\eta_{0} \bowtie_{0} \eta_{1} \ldots \bowtie_{k-1} \eta_{k} \ldots
$$

With this adjustment, $\left.v^{*}\right|_{s}$ (for $s>n+2 m$ ) will not appear in $\Phi_{\epsilon}\left(\eta_{i}\right)$ because $d\left(u^{*}, \overline{O_{f}\left(v^{*}\right)}\right)>8 \epsilon$. (By $\Phi_{\epsilon}\left(\eta_{i}\right)$ we mean any orbit of length $\left|\eta_{i}\right|$ that $\epsilon$-shadows the pieces in $\eta_{i}$ with $\smile$ omitted.) Let $n_{k}^{\prime}=\left|\eta_{k}\right|, m_{k}=\left|\bowtie_{k}\right|$ and $\ell_{k}=\left|\eta_{0} \bowtie_{0} \ldots \eta_{k-1} \bowtie_{k-1}\right|$. Then, by (32), we have $n_{k}^{\prime}=O\left(k^{4} e^{k^{4}}\right) \gg m_{k}$.

Next, we define new elements $a, b$ and $w_{0}$ like those in $\$ 5.2$ :

$$
a=\smile u^{*} \smile u^{*} \smile \cdots u^{*} \smile \text { with }|a|>n+4(2 m+1) \text {; }
$$

(ii) $\quad b=\left.\smile u^{*} \smile v^{*}\right|_{\hat{s}} \smile u^{*} \smile$ with $|a|=|b|$;

(iii) $w_{0}=\left.u^{*} \smile v^{*}\right|_{\hat{r}} \smile u^{*} \smile$ with $\hat{r}>\max \left\{k_{0},|a|\right\}$, where $k_{0}$ is as in (32).

Let $q_{-1}=0, p_{-1}^{\prime \prime}=\left|w_{0}\right|-|v|-m$ and

$$
\begin{aligned}
& p_{k}^{\prime \prime}=p_{k}+\sum_{q_{j}<p_{k}}\left(p_{j}^{\prime \prime}+|v|+m\right)+\sum_{r_{j}^{\prime}<p_{k}}|v|, \\
& q_{k}^{\prime \prime}=q_{k}+\sum_{q_{j}<q_{k}}\left(p_{j}^{\prime \prime}+|v|+m\right)+\sum_{r_{j}^{\prime}<q_{k}}|v|,
\end{aligned}
$$

where $r_{j}^{\prime}$ is to be constructed as in $\$ 5.2$, counting the position at which we insert the segment $b$. (The term $m$ in the first sum of both (34) and (35) corresponds to the extra $\smile$ in (36) below.)

Write $\mathbf{y}=\sigma_{0} \sigma_{1} \ldots \sigma_{k} \ldots$ with $\left|\sigma_{0} \ldots \sigma_{k}\right|=q_{k}$. In the following, we define $\left\{\sigma_{k}^{\prime}\right\}$ and $\left\{\mathbf{y}_{k}\right\}$.

(i) For

$$
\sigma_{k+1}=\eta_{s}^{T} \bowtie_{s} \eta_{s+1} \ldots \bowtie_{t-1} \eta_{t}^{T}
$$

as in (26), we modify it to $\sigma_{k+1}^{\prime}$ so that no segment of $\sigma_{k+1}^{\prime}$ contains $\mathbf{z}=\left.\mathbf{y}_{k}\right|_{p_{k+1}^{\prime \prime}}$ (analogous to what was done in §5.2). For $s+1 \leq i \leq t$, since $u^{*}$ appears in every $n+2 m+1$ symbols of $\eta_{i}$, if a segment of $O_{f}\left(v^{*}\right)$ with length $n+2 m+1$ already appears in $S^{m_{i-1}}(\mathbf{z})$ (not counting the $\smile$ and $\bowtie_{t}$ in $\mathbf{z}$ ), then, by observing $d\left(u^{*}, \overline{O_{f}\left(v^{*}\right)}\right)>8 \epsilon$, the requirement is fulfilled; in this case we can just take $\eta_{i}^{\prime}=\eta_{i}$ and set $r_{i}^{\prime}=\infty$. Otherwise, we let

$$
\eta_{i}^{\prime}=\eta_{i}(0) \ldots \eta_{i}(j-1) b \eta_{i}(j) \ldots \eta_{i}\left(n_{i}^{\prime}-1\right)
$$

and $r_{i}^{\prime}=\ell_{i}+j$, where $j$ is such that $u^{*}$ appears in every $n+2 m+1$ symbols of

$$
\mathbf{z}(\xi+j) \ldots \mathbf{z}(\xi+j+|v|-1) \quad \text { for each } \xi \leq m_{i-1} .
$$


(ii) We define $\mathbf{y}_{k+1}=\mathbf{y}_{k} w_{k+1} \sigma_{k+1}^{\prime}$, where

$$
w_{k+1}= \begin{cases}\left.\smile\left(\mathbf{y}_{k}\right)\right|_{p_{k}^{\prime \prime}} a & \text { if } p_{k} \leq q_{i}<p_{k+1} \text { for some } i, \\ \left.\smile\left(\mathbf{y}_{k}\right)\right|_{p_{k}^{\prime \prime}} b & \text { otherwise. }\end{cases}
$$

Note that the above $\mathbf{y}_{k}$ is not necessarily a segment of an orbit. Hence, to complete the construction, we need to make a further refinement:

$$
w_{k+1}^{\prime}= \begin{cases}\left.\smile \Phi\left(\mathbf{y}_{k}\right)\right|_{p_{k}^{\prime \prime}} a & \text { if } p_{k} \leq q_{i}<p_{k+1} \text { for some } i, \\ \left.\smile \Phi\left(\mathbf{y}_{k}\right)\right|_{p_{k}^{\prime \prime}} b & \text { otherwise, }\end{cases}
$$

where $\Phi$ is the shadowing function defined in (iii) at the end of $\S 3$. (We make the convention that once $\eta_{i}^{\prime}$ is determined, so are $\Phi_{\epsilon}\left(\eta_{i}^{\prime}\right)$ and $\Phi_{\left\{\epsilon_{i}\right\}}\left(\eta_{0}^{\prime} \bowtie_{0} \ldots \bowtie_{i-1} \eta_{i}^{\prime}\right)$.) Now let

$$
G(h(\mathbf{i}))=w_{0} \sigma_{0}^{\prime} w_{1}^{\prime} \sigma_{1}^{\prime} w_{2}^{\prime} \sigma_{2}^{\prime} \ldots w_{k}^{\prime} \sigma_{k}^{\prime} \ldots,
$$

and let $x_{\mathbf{i}}^{\prime}=\Phi(G(h(\mathbf{i})))$. We collect all the $x_{\mathbf{i}}^{\prime}$ with $\mathbf{i} \in Q$ in Construction $\mathrm{M}$ and denote this set by $F^{\prime}$. We will see, in Lemma 5.8, that $F^{\prime}$ is a Moran fractal.

The main theorem follows from the following lemmas. As before, we need conditions on $\left\{p_{k}\right\}$ and $\left\{q_{k}\right\}$ to ensure the recurrence property. The proof is analogous to the arguments for Lemmas 5.1 and 5.3 and hence is omitted.

LEMMA 5.5. For any $0 \leq \alpha \leq \beta \leq \infty$, there exist $\left\{p_{k}\right\}_{k=0}^{\infty}$ and $\left\{q_{k}\right\}_{k=0}^{\infty}$, with $p_{k+1}<q_{k}$, which satisfy (for each $k$ )

$$
\begin{gathered}
2 M<p_{k}-p_{k-1}, \\
\ell_{i}+2 M<p_{k}<\ell_{i}+n_{i}^{\prime}-2 M \quad \text { for some } i, \\
\text { and } \ell_{j}+4 p_{k}<q_{k}<\ell_{j}+n_{j}^{\prime}-4 p_{k} \text { for some } j,
\end{gathered}
$$

where $M=n+2 m+1$; the sequences $\left\{p_{k}^{\prime \prime}\right\}_{k=0}^{\infty},\left\{q_{k}^{\prime \prime}\right\}_{k=0}^{\infty}$ defined by (34) and (35) satisfy $p_{k}^{\prime}<3 p_{k}$ and (18)-(20).

The selection of $p_{k}, q_{k}$ is after the construction of $G_{1}(\cdot)$. We may take, for example, $p_{k}=O(3 k M)$ so that each $p_{k}$ is the end position of a piece of length $n$ in (33), and then omit some items and relabel the sequence so that (38) holds. Take $q_{k}$ (which depends on $\alpha, \beta)$ as in the proof of Lemma 5.3 (see Appendix A); then adjust the sequence to satisfy (39). We may assume that each $q_{k}$ avoids the places of $\smile$. Hence the construction will not disturb the $\smile$ in the process of cutting and inserting the recurrence pieces.

We now consider the recurrence behavior of the $G(h(\mathbf{i}))$ in (37). Let $S$ be the (left) shift map on $X^{\mathbb{N}}$. For $x, x^{\prime} \in X^{\mathbb{N}}$, we let

$$
\mathbf{d}_{n}\left(x, x^{\prime}\right):=\max \left\{d\left(x(i), x^{\prime}(i)\right): i=0,1, \ldots, n-1\right\},
$$

where $d$ is the metric on $X$. Note that $\mathbf{d}_{n}$ is a semi-metric on $X^{\mathbb{N}}$.

LEMMA 5.6. Let $z=G(h(\mathbf{i}))$ as in (37). Then for any $k \in \mathbb{N}$, we have

$$
\begin{aligned}
& \mathbf{d}_{p_{k}^{\prime \prime}}\left(z, S^{i}(z)\right)>5 \epsilon \quad \text { for all } m<i<q_{k}^{\prime \prime}, \\
& \mathbf{d}_{p_{k}^{\prime \prime}}\left(z, S^{i}(z)\right) \leq \epsilon+\epsilon_{0} \quad \text { for } i=q_{k}^{\prime \prime}+m .
\end{aligned}
$$


Proof. Let $z^{\prime}=w_{0} \sigma_{0}^{\prime} w_{1} \sigma_{1}^{\prime} w_{2} \sigma_{2}^{\prime} \ldots w_{k} \sigma_{k}^{\prime} \ldots \in X^{\mathbb{N}}$ be the sequence corresponding to $z$. By the same argument as in Proposition 5.4, we obtain that for any $m<i<q_{k}^{\prime \prime}$, there exists some $j<p_{k}^{\prime \prime}$ such that

$$
\begin{aligned}
\text { either } z^{\prime}(j) & =u^{*}, \quad\left(S^{i}\left(z^{\prime}\right)\right)(j) \in O_{f}\left(v^{*}\right), \\
\text { or } z^{\prime}(j) & \in O_{f}\left(v^{*}\right), \quad\left(S^{i}\left(z^{\prime}\right)\right)(j)=u^{*} .
\end{aligned}
$$

Recall that $d\left(u^{*}, \overline{O_{f}\left(v^{*}\right)}\right)>8 \epsilon$. This implies

$$
\mathbf{d}_{p_{k}^{\prime \prime}}\left(z^{\prime}, S^{i}\left(z^{\prime}\right)\right)>8 \epsilon \text { for all } m<i<q_{k}^{\prime \prime} .
$$

From the definition of map $\Phi$, we see that

$$
\mathbf{d}_{\left|w_{k}\right|}\left(w_{k}, w_{k}^{\prime}\right) \leq \epsilon+\epsilon_{0} .
$$

(Here we assume that $w_{k}^{\prime}$ also $\left(\epsilon+\epsilon_{0}\right)$-shadows $\smile$ and $\bowtie_{k}$ of $w_{k}$ in the corresponding positions. This will not affect the following argument as (40) is deduced from $d\left(u^{*}, \overline{O_{f}\left(v^{*}\right)}\right)>8 \epsilon$.) Hence

$$
\mathbf{d}_{l}\left(z, z^{\prime}\right) \leq \epsilon+\epsilon_{0} \quad \text { for any } l \in \mathbb{N}
$$

and the first statement of the lemma follows by (40). Equation (41) implies the second statement by observing that $\mathbf{d}_{p_{k}^{\prime \prime}}\left(z^{\prime}, S^{i}\left(z^{\prime}\right)\right)=0$ for $i=q_{k}^{\prime \prime}+m$.

As a consequence of Lemma 5.6, we have the following.

LEMMA 5.7. $F^{\prime}$ is a subset of $D(\alpha, \beta)$.

Proof. Let $x_{\mathbf{i}}^{\prime} \in F^{\prime}$. It cannot be a point with period less than $m+1$, owing to the insertion of $u^{*}$ and $\left.v^{*}\right|_{\hat{r}},\left.v^{*}\right|_{\hat{s}}$. Thus there exists $0<\epsilon^{\prime}<\epsilon$ such that

$$
\tau_{B\left(x_{\mathbf{i}}^{\prime}, \epsilon^{\prime}\right)}\left(x_{\mathbf{i}}^{\prime}\right)=\min \left\{j>0: f^{j}\left(x_{\mathbf{i}}^{\prime}\right) \in B\left(x_{\mathbf{i}}^{\prime}, \epsilon^{\prime}\right)\right\}>m .
$$

Consider $\tau_{B\left(x_{\mathbf{i}}^{\prime}, \epsilon^{\prime}\right)}\left(x_{\mathbf{i}}^{\prime}\right)$. By the definition of $\Phi$, the orbit of $x_{\mathbf{i}}^{\prime}$ is within $\epsilon+\epsilon_{0}(<5 \epsilon / 4)$ distance of the pieces (excluding the $\smile$ and $\bowtie_{k}$ ) of $G(h(\mathbf{i})$ ) in the corresponding places. Hence we have by Lemma 5.6 that

$$
\tau_{B_{p_{k}^{\prime \prime}}\left(x_{\mathbf{i}}^{\prime}, \epsilon^{\prime}\right)}\left(x_{\mathbf{i}}^{\prime}\right) \geq q_{k}^{\prime \prime} \quad \text { for all } k \in \mathbb{N} .
$$

Similarly, we obtain from Lemma 5.6 that

$$
\tau_{B_{p_{k}^{\prime \prime}}\left(x_{\mathbf{i}}^{\prime}, 4 \epsilon\right)}\left(x_{\mathbf{i}}^{\prime}\right) \leq q_{k}^{\prime \prime}+m \quad \text { for all } k \in \mathbb{N} .
$$

Combining the two estimates, we conclude by Lemma 5.5 and Proposition 2.6 that

$$
\underline{\tau}\left(x_{\mathbf{i}}^{\prime}\right)=\alpha \quad \text { and } \quad \bar{\tau}\left(x_{\mathbf{i}}^{\prime}\right)=\beta,
$$

which proves the lemma.

Our last step is to estimate the topological entropy of $F^{\prime}$.

LEMMA 5.8. $F^{\prime}$ is a dynamically defined Moran fractal and

$$
h_{t}\left(f, F^{\prime}\right) \geq \frac{n}{n+2 m+1} h_{t}(f, F) .
$$

\section{CAmbridge JDUNALS}


Proof. For the $s$ th element of $\left\{z_{i j}\right\}_{i j}$ in $h(\mathbf{i})$ (counting lexically) in (31), we let $l_{s}, l_{s}^{\prime}$ and $l_{s}^{\prime \prime}$ be its positions in $h(\mathbf{i}), G_{1}(h(\mathbf{i}))$ and $G_{1}(h(\mathbf{i}))$, respectively. Then

$$
l_{s}^{\prime}= \begin{cases}\sum_{i=0}^{k}\left(n_{i}^{\prime}+m_{i}\right)-m_{k} & \text { if } s=t_{k} \text { for some } k, \\ \sum_{i=0}^{k}\left(n_{i}^{\prime}+m_{i}\right)+c\left(\frac{n_{k+1}\left(s-t_{k}\right)}{n}-1\right) & \text { if } t_{k}<s<t_{k+1},\end{cases}
$$

where

$$
\begin{gathered}
t_{k}=\sum_{i=0}^{k} N_{i}-1, \quad n_{i}^{\prime}=c\left(\frac{n_{i} N_{i}}{n}-1\right) \quad \text { and } \quad c=n+2 m+1 ; \\
l_{s}^{\prime \prime}=l_{s}^{\prime}+\sum_{q_{k}<l_{s}^{\prime}} p_{k}^{\prime \prime} \quad \text { for all } s \in \mathbb{N} .
\end{gathered}
$$

It is clear that $l_{s}<l_{s}^{\prime} \leq(n+2 m+1 / n) l_{s}$. Moreover, since $\lim _{k \rightarrow \infty} \sum_{i=1}^{k} p_{i}^{\prime \prime} / q_{k}=0$, we have $\lim _{s \rightarrow \infty} l_{s}^{\prime} / l_{s}^{\prime \prime}=1$. It follows that $\underline{\lim }_{s \rightarrow \infty} l_{s} / l_{s}^{\prime \prime} \geq n /(n+2 m+1)$.

Next, take $\mathbf{i}=\mathbf{i}_{0} \mathbf{i}_{1} \ldots \in Q$ with $\left|\mathbf{i}_{i}\right|=N_{i}$, and write

$$
G(h(\mathbf{i})):=\widetilde{\eta}_{0} \bowtie_{0} \tilde{\eta}_{1} \bowtie_{1} \ldots \bowtie_{k-1} \tilde{\eta}_{k} \ldots,
$$

where each $\tilde{\eta}_{i}$ is a tuple of elements of $\mathcal{X}$ connected by $\smile$. For $k \in \mathbb{N}$, we let $y_{\mathbf{i}_{k}}=\Phi_{\epsilon}\left(\tilde{\eta}_{k}\right)$ and

$$
x_{\mathbf{i}_{0} \ldots \mathbf{i}_{k}}^{\prime}=\Phi_{\left\{\epsilon_{i}\right\}}\left(y_{\mathbf{i}_{0}}, \ldots, y_{\mathbf{i}_{k}}\right) .
$$

For any $i_{0} \ldots i_{s} \in Q_{s}$, we let

$$
\Delta_{i_{0} \ldots i_{s}}= \begin{cases}\overline{B_{l_{t_{k}}^{\prime \prime}}\left(x_{i_{0} \ldots i_{t_{k}}}^{\prime}, 2 \epsilon_{k}\right)} & \text { if } s=t_{k}, \\ \bigcup_{i_{s+1}, \ldots, i_{t_{k}}} \Delta_{i_{0} \ldots i_{t_{k}}} & \text { if } t_{k-1}<s<t_{k} .\end{cases}
$$

Then it is easy to check that $F^{\prime}$ is a dynamically defined Moran fractal modeled by $Q$ with the level sets $\Delta_{i_{0} \ldots i_{s}}$ defined above. Moreover, the set $F^{\prime}$ has entropy

$$
h_{t}\left(f, F^{\prime}\right)=\varliminf_{s \rightarrow \infty} \frac{1}{l_{s}^{\prime \prime}} \log \# Q_{s} .
$$

Consequently, we have

$$
h_{t}\left(f, F^{\prime}\right) \geq \lim _{s \rightarrow \infty} \frac{l_{s}}{l_{s}^{\prime \prime}} h_{t}(f, F) \geq \frac{n}{n+2 m+1} h_{t}(f, F) .
$$

Proof of Theorem 1.1. Let $0 \leq \alpha \leq \beta \leq \infty$. Let $F$ be a Moran fractal as in Proposition 4.6. Choose $\left\{p_{k}\right\}_{k},\left\{q_{k}\right\}_{k}$ as in Lemma 5.5, and let $F^{\prime}$ be the reconstruction of $F$ in the above. We have by Lemma 5.7 that

$$
h_{t}(f, D(\alpha, \beta)) \geq h_{t}\left(f, F^{\prime}\right) .
$$


For any $\vartheta>0$, by taking $n$ to be large compared with $m$, Lemma 5.8 implies

$$
h_{t}\left(f, F^{\prime}\right) \geq h_{t}(f, F)-\vartheta=h_{t}(f)-\vartheta .
$$

It follows that $h_{t}(f, D(\alpha, \beta)) \geq h_{t}(f)-\vartheta$. This proves $h_{t}(f, D(\alpha, \beta))=h_{t}(f)$, since $\vartheta>0$ is arbitrary.

For any non-empty open set $U \subseteq X$, we choose $z^{*}$ with $B_{t}\left(z^{*}, 5 \epsilon\right) \subseteq U$ for some $t \in \mathbb{N}$. If we replace $w_{0}$ in the construction of $F^{\prime}$ by

$$
\left.\left.z^{*}\right|_{t} \smile u^{*} \smile v^{*}\right|_{\hat{r}} \smile u^{*} \smile \quad \text { for some large } \hat{r},
$$

then the new Moran fractal denoted by $F^{\prime \prime}$ will be a subset of $U \cap D(\alpha, \beta)$. The conclusion that $h_{t}(f, U \cap D(\alpha, \beta))=h_{t}(f)$ follows from the same argument as for $D(\alpha, \beta)$ in the previous paragraph.

Acknowledgements. The authors would like to express their gratitude to Professors Aihua Fan and Dejun Feng for many valuable suggestions and comments during the preparation of this paper. The second author thanks Professor Ercai Chen for pointing out reference [15]. This paper is part of the second author's $\mathrm{PhD}$ thesis; she is grateful to the Mathematics Department of CUHK for providing support to carry out the research.

This research was partially supported by a HKRGC grant.

\section{A. Appendix}

We begin with the following lemma.

LemmA A.1. Let $0 \leq \alpha \leq \beta \leq \infty$ and let $C>1$. For any strictly increasing sequence $\left\{p_{k}\right\}_{k=0}^{\infty}$ with $\lim _{k \rightarrow \infty} p_{k} / p_{k-1}=1$. there exists $\left\{q_{k}\right\}_{k=0}^{\infty}$ such that

$$
\begin{gathered}
C \cdot p_{k}<q_{k}-q_{k-1}, \\
e^{\sqrt{p_{k}}}<q_{k}<e^{p_{k}^{2}} \quad \text { for large } k, \\
\text { and } \quad \varlimsup_{k \rightarrow \infty}\left(\log q_{k}\right) / p_{k}=\alpha, \quad \varlimsup_{k \rightarrow \infty}\left(\log q_{k}\right) / p_{k}=\beta .
\end{gathered}
$$

The proof is the same as in $\left[\mathbf{1 5}, \mathrm{p}\right.$. 84] for the case $p_{k}=k$. Basically, the condition $0 \leq \alpha \leq \beta \leq \infty$ is divided into eight cases according to whether each relation is $<$ or $=$. For example, when $0<\alpha=\beta<\infty$, one can take $q_{k}=\left[e^{\alpha k}\right]$ (where $[a]$ denotes the integrer part of $a$ ); if $0<\alpha<\beta<\infty$, one can take $q_{k}=\sum_{i=1}^{k} u_{i}$, where

$$
u_{k}= \begin{cases}{\left[e^{\alpha k}\right]} & \text { if } k \in \mathcal{K}, \\ {\left[e^{\beta k}\right]} & \text { otherwise }\end{cases}
$$

and $\mathcal{K}=\left\{k \in \mathbb{N}: \sum_{j=1}^{2 i-1} 2^{4^{j}} \leq k<\sum_{j=1}^{2 i} 2^{4^{j}}\right.$ for some integer $\left.i>0\right\}$.

Proof of Lemma 5.1. Let $\left\{p_{k}\right\}_{k=0}^{\infty}$ and $\left\{q_{k}\right\}_{k=0}^{\infty}$ be as in Lemma A.1. To prove the lemma, it suffices to show that $\lim _{k \rightarrow \infty} p_{k}^{\prime} / p_{k}=1$ and $\lim _{k \rightarrow \infty} q_{k}^{\prime} / q_{k}=1$. Take $p_{0}>3$. We first claim that $p_{k}^{\prime} \leq 3 p_{k}$. This will be proved by induction on the $q_{i}$. It is clear that the statement is true for $p_{k} \leq q_{1}$, since $p_{k}^{\prime} \leq p_{k}+p_{0}+5$ for such $k$. Now assume that the statement holds for $p_{k} \leq q_{i}$. 


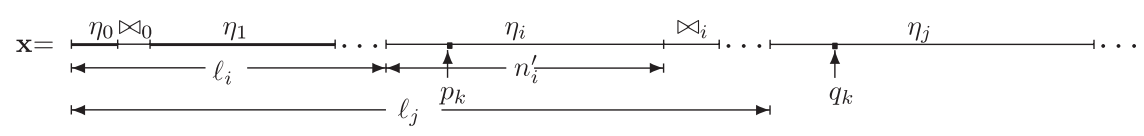

FIGURE A.1. The position of $p_{k}, q_{k}$ in Lemma 5.3.

For any $k$ such that $q_{i}<p_{k} \leq q_{i+1}(1 \leq i)$, we let $j$ be such that $p_{j} \leq q_{i-1}<p_{j+1}$. Then

$$
\begin{aligned}
p_{k}^{\prime} & =p_{j}^{\prime}+p_{k}-p_{j}+p_{i-1}^{\prime}+1+p_{i}^{\prime}+1 \\
& \leq 2 p_{j}+p_{k}+3 p_{i-1}+3 p_{i}+2 \quad \text { by }(A .1) \\
& \leq 2 p_{j}+p_{k}+2\left(q_{i}-q_{i-1}\right) \\
& \leq 2 p_{j}+p_{k}+2\left(p_{k}-p_{j}\right)=3 p_{k} .
\end{aligned}
$$

This proves the claim. We may assume (A.2) holds for all $k \geq 0$ by taking $p_{0}$ large. Then, by using $p_{j}^{\prime} \leq 3 p_{j}$ and $e^{\sqrt{p_{j}}} \leq q_{j}$, we have

$$
\sum_{i=-1}^{k} p_{i}^{\prime} \leq \sum_{i=-1}^{k} 3 p_{i} \leq 9 p_{k}^{2} \leq 9 \log q_{k}
$$

and hence $\lim _{k \rightarrow \infty} \sum_{i=-1}^{k} p_{i}^{\prime} / q_{k}=0$. Since $e^{\sqrt{p_{j}}} \leq q_{j}, q_{j}<p_{k}$ implies $p_{j} \leq\left(\log p_{k}\right)^{2}$. Therefore, by using $p_{j}^{\prime} \leq 3 p_{j}$, we have

$$
\begin{aligned}
p_{k} \leq p_{k}^{\prime} & =p_{k}+\sum_{q_{j}<p_{k}}\left(p_{j}^{\prime}+1\right) \\
& \leq p_{k}+\sum_{p_{j} \leq\left(\log p_{k}\right)^{2}}\left(3 p_{j}+1\right) \\
& \leq p_{k}+9\left(\log p_{k}\right)^{4}
\end{aligned}
$$

and so $\lim _{k \rightarrow \infty} p_{k}^{\prime} / p_{k}=1$. Similarly, we have

$$
q_{k} \leq q_{k}^{\prime}=q_{k}+\sum_{i=-1}^{k-1}\left(p_{j}^{\prime}+1\right) \leq q_{k}+9 p_{k}^{2},
$$

which implies $\lim _{k \rightarrow \infty} q_{k}^{\prime} / q_{k}=1$. Thus (20) follows.

Proof of Lemma 5.3. Let $\left\{p_{k}\right\}_{k=0}^{\infty}$ be a strictly increasing sequence such that $\lim _{k \rightarrow \infty} p_{k} / p_{k-1}=1$ and $p_{k} \leq e^{\sqrt{p_{k-1}}}$. Take $n_{k}^{\prime}=O\left(\left[e^{k^{4}}\right]\right)$ and $m_{k}=k$, so that $n_{k}^{\prime} \gg m_{k}$. We may assume (29) for the sequence, since $\lim _{k \rightarrow \infty} m_{k} / \ell_{k+1}=0$. Next, let $\left\{q_{k}\right\}_{k=0}^{\infty}$ be as in Lemma A.1 with $C=15$. (See Figure A.1 for the position of $p_{k}, q_{k}$.) By taking $p_{0}$ to be large, we assume $e^{\sqrt{p_{k}}}<q_{k}<e^{p_{k}^{2}}$. Increase $q_{k}$ if necessary so that (30) holds; then we find $\left\{q_{k}\right\}_{k=0}^{\infty}$ which satisfies (A.3), (30) and is such that

$$
\begin{gathered}
6 p_{k}<q_{k}-q_{k-1}, \\
e^{\sqrt{p_{k}}}<q_{k}<c e^{p_{k}^{2}} \text { for some } c>0 .
\end{gathered}
$$

\section{CAMBridge JOURNALS}


Let $\left\{p_{k}^{\prime}\right\}_{k=0}^{\infty},\left\{q_{k}^{\prime}\right\}_{k=0}^{\infty}$ be defined by (24), (25). Recall that $r_{j}$ is defined to be infinity or at the beginning position in each $\eta_{j}$. Let $s_{k}=\max \left\{i: \ell_{i}<p_{k}\right\}$ and $t_{k}=\max \left\{i: \ell_{i}<q_{k}\right\}$. We have $p_{0}^{\prime}<p_{0}+m_{s_{0}-1}$ (where $m_{s_{0}-1}$ is the length of $w_{0}=b b \ldots b$ if $\sigma_{0}$ contains $b b$ ) and

$$
\begin{gathered}
p_{k}^{\prime} \leq p_{k}+\sum_{q_{j}<p_{k}}\left(p_{j}^{\prime}+1\right)+s_{k}-s_{0}, \\
q_{k}^{\prime} \leq q_{k}+\sum_{q_{j}<q_{k}}\left(p_{j}^{\prime}+1\right)+t_{k} .
\end{gathered}
$$

Hence, as in Lemma 5.1, we can show $p_{k}^{\prime} \leq 3 p_{k}$ by using (A.4) and by observing that $s_{j}-s_{i} \leq p_{j}-p_{i}$ for $i<j$. The arguments for showing $\lim _{k \rightarrow \infty} \sum_{i=-1}^{k} p_{i}^{\prime} / q_{k}=0$, $\lim _{k \rightarrow \infty} p_{k}^{\prime} / p_{k}=1$ and $\lim _{k \rightarrow \infty} q_{k}^{\prime} / q_{k}=1$ are also the same as for Lemma 5.1. Then (18)-(20) follow.

LemmA A.2. For any $i$ with $\ell_{i} \leq q_{k+1}$, we have $m_{i-1}<\sqrt{p_{k+1}}$. The expression (28) holds for some $j$.

Proof. Note that $q_{k}<e^{p_{k}^{2}}$. If $p_{0}$ is large, then for any $i$ with $\ell_{i} \leq q_{k+1}$ we have $m_{i-1}<$ $\sqrt{p_{k+1}}$, and for any $t$ with $\ell_{t}<p_{k+1}$ we have $m_{t}<\left(p_{k+1}\right)^{1 / 4}$. The total length of $\bowtie_{t}$ 's contained in $\mathbf{z}$ is less than $\Sigma_{\ell_{t}<p_{k+1}} m_{t}$. Hence

$$
p_{k+1}-2 m_{i-1}-\sum_{\ell_{t}<p_{k+1}}\left(m_{t}+m_{i-1}\right) \geq p_{k+1}-3 \sqrt{p_{k+1}} \geq 1 .
$$

Then there exists some $j$ with $m_{i-1}<j<p_{k+1}-m_{i-1}$ such that $\mathbf{z}(j+l)$ does not belong to any $\bowtie_{t}$ of $\mathbf{z}$. Since the segment $S^{m_{i-1}}(\mathbf{z})$ (not counting $\bowtie_{t}$ 's in $\mathbf{z}$ ) does not contain the letter $b$, we must have that $j$ satisfies (28).

Proof of Proposition 5.4. Take sequences $\left\{p_{k}\right\}_{k=0}^{\infty}$ and $\left\{q_{k}\right\}_{k=0}^{\infty}$ as in Lemma 5.3. We can express $\mathbf{y} \in \Lambda^{\prime}(F)$ as

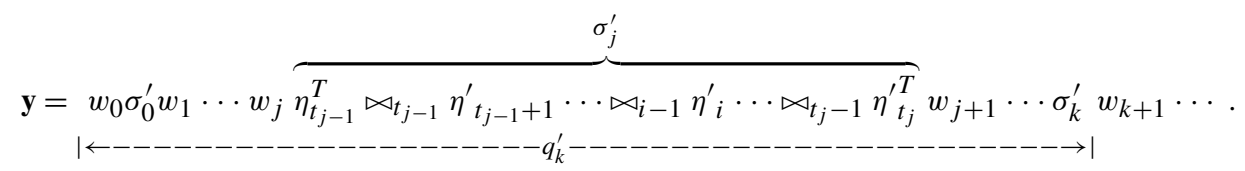

(Here $t_{j}$ is such that $q_{j}$ belongs to the piece $\eta_{t_{j}}$.) We will show that $R_{k}(\mathbf{y})=q_{k}^{\prime}$. First note that $\left|w_{k+1}\right|=p_{k}^{\prime}+1$. It is clear that if $n=q_{k}^{\prime}$, then

$$
\left.S^{n}(\mathbf{y})\right|_{p_{k}^{\prime}}=\left.w_{k+1}\right|_{p_{k}^{\prime}}=\left.\mathbf{y}\right|_{p_{k}^{\prime}} .
$$

On the other hand, for $n<q_{k}^{\prime}$ we have $\left.S^{n}(\mathbf{y})\right|_{p_{k}^{\prime}} \neq\left.\mathbf{y}\right|_{p_{k}^{\prime}}$ by the following observations.

(i) $\mathbf{y}(n)=\sigma_{j}^{\prime}(r)$ for $j \leq k$ and $r<\left|\sigma_{j}^{\prime}\right|$ implies that $S^{n}(\mathbf{y})(0)=\sigma_{j}(r) \in \mathcal{A} \neq y(0) \in \mathcal{A}^{c}$ if $t_{j-1}=t_{j}$, or (in the case where $\left.t_{j-1}<t_{j}\right)$ that $\left.\mathbf{y}\right|_{p_{j}^{\prime}}=\left.S^{m}\left(\triangleleft_{i-1} \eta_{i}^{\prime}\right)\right|_{p_{j}^{\prime}}\left(\right.$ or $S^{m}\left(\bowtie_{t_{j}-1}\right.$ $\left.\eta_{t_{j}}^{\prime T}\right)\left.\right|_{p_{j}^{\prime}}$ ) for some $t_{j-1}<i<t_{j}$ and $m<m_{i-1}$. This is because the first two letters of $\left.\mathbf{y}\right|_{p_{j}^{\prime}}$ are $b b$, but this does not appear in $\eta_{i}^{\prime}, \eta_{t_{j-1}}^{T}$ or $\eta_{t_{j}}^{\prime T}$ (note that $\eta_{t_{j}}^{T}$ does not end with $b$ ). However, by construction of $\eta_{i}^{\prime}$, we have either that $\eta_{i}^{\prime}=\eta_{i} \in \mathcal{A}_{*}$ and $S^{m_{i-1}}\left(\left.\mathbf{y}\right|_{p_{j}^{\prime}}\right)$ contains the letter $b \in \mathcal{A}^{c}$, or that

$$
\eta_{i}^{\prime}(t)=b \in \mathcal{A}^{c} \neq \mathbf{y}\left(t+m_{i-1}-m\right) \in \mathcal{A} \quad \text { where } t=r_{i}-\ell_{i},
$$

by (27) and (28). Thus the desired contradiction is obtained. 
(ii) $\mathbf{y}(n)=w_{j}(0)$ for $j \leq k$ implies that $\left.S^{n+n^{\prime}}(\mathbf{y})\right|_{l}=\left.S^{n^{\prime}}(\mathbf{y})\right|_{l} \neq\left. S^{n^{\prime}}\left(w_{j} \sigma_{j}^{\prime}\right)\right|_{l}$ for $n^{\prime}=p_{j-1}^{\prime}$ and $l=p_{j}^{\prime}-p_{j-1}^{\prime}$. This is because if $l=p_{j}-p_{j-1}$, then $\mathbf{x}_{j}\left(p_{j-1}^{\prime}\right) \in \mathcal{A} \neq$ $w_{j}\left(p_{j-1}^{\prime}\right)=b \in \mathcal{A}^{c} ;$ otherwise, $\mathbf{x}_{j}\left(p_{j-1}^{\prime}\right) \ldots \mathbf{x}_{j}\left(p_{j}^{\prime}-1\right) \notin \mathcal{A}_{*} \neq\left.\left(a \sigma_{j}^{\prime}\right)\right|_{l} \in \mathcal{A}_{*}$.

(iii) $\mathbf{y}(n)=w_{j}\left(p_{j-1}^{\prime}\right)$ for $j \leq k$ implies that $\left.S^{n}(\mathbf{y})\right|_{2}=b b \neq w_{j}\left(p_{j-1}^{\prime}\right) \sigma_{j}(0)$, since $\sigma_{j}(0) \in \mathcal{A}$.

(iv) $\mathbf{y}(n)=w_{j}(i)$ for $i=1$ or $p_{j-1}^{\prime}-1$ and $j \leq k$ implies that $S^{n}(\mathbf{y})(1)=w_{j}(2) \in \mathcal{A} \neq$ $\mathbf{y}(1)=b \in \mathcal{A}^{c}$, or $S^{n}(\mathbf{y})(0)=w_{j}(i) \in \mathcal{A} \neq \mathbf{y}(0) \in \mathcal{A}^{c}$.

(v) $\mathbf{y}(n)=w_{j}(i)$ for $p_{j-1} \leq q_{0}, 1<i<p_{j-1}^{\prime}-1$ implies that $S^{n}(\mathbf{y})(0)=w_{j}(i) \in$ $\mathcal{A} \neq \mathbf{y}(0) \in \mathcal{A}^{c}$.

(vi) $\mathbf{y}(n)=w_{j}(i)$ for $q_{r-1}<p_{j-1} \leq q_{r}, 1<i<p_{j-1}^{\prime}-1$ and $r<j \leq k$ implies that

$$
\left.\mathbf{y}\right|_{p_{j-1}^{\prime}}=\left.S^{n}(\mathbf{y})\right|_{p_{j-1}^{\prime}}=\left.S^{i}\left(w_{j} \sigma_{j}\right)\right|_{p_{j-1}^{\prime}} .
$$

If $i<q_{0}^{\prime}$, then $\mathbf{y}(0) \in \mathcal{A}^{c} \neq w_{j}(i) \in \mathcal{A}$; and if $i>p_{j-1}^{\prime}-q_{r-1}^{\prime}$, then $\mathbf{y}\left(q_{r-1}^{\prime}\right) \in \mathcal{A}^{c} \neq$ $\sigma_{j}(s) \in \mathcal{A}$ for $s=i+q_{r-1}^{\prime}-p_{j-1}^{\prime}+1$. Consequently, (A.5) holds for $q_{0}^{\prime} \leq i \leq p_{j-1}^{\prime}-$ $q_{r-1}^{\prime}$. Note that $\left.\mathbf{y}\right|_{p_{j-1}^{\prime}}=\left.w_{j}\right|_{p_{j-1}^{\prime}}$. It follows that

$$
\left.\mathbf{y}\right|_{l}=\left.S^{i}(\mathbf{y})\right|_{l} \quad \text { for } l=p_{j-1}^{\prime}-i .
$$

This implies $\left.\mathbf{y}\right|_{p_{r}^{\prime}}=\left.S^{m}(\mathbf{y})\right|_{p_{r}^{\prime}}$ for some $m<q_{r}^{\prime}$. Repeat the above argument for $\left.\mathbf{y}\right|_{p_{r}^{\prime}}$. By virtue of (v), we must arrive at a contradiction in a finite number of steps.

Since $\lim _{k \rightarrow \infty} p_{k}^{\prime} / p_{k-1}^{\prime}=1$ by (19), the proof is complete upon using $R_{k}(\mathbf{y})=q_{k}^{\prime}$ and Proposition 2.6.

\section{REFERENCES}

[1] L. M. Barreira. A non-additive thermodynamic formalism and applications to dimension theory of hyperbolic dynamical systems. Ergod. Th. \& Dynam. Sys. 16 (1996), 871-927.

[2] L. Barreira and B. Saussol. Hausdorff dimension of measures via Poincaré recurrence. Comm. Math. Phys. 219 (2001), 443-463.

[3] L. Barreira and B. Saussol. Product structure of Poincaré recurrence. Ergod. Th. \& Dynam. Sys. 22 (2002), 33-61.

[4] F. Blanchard. $\beta$-Expansions and symbolic dynamics. Theoret. Comput. Sci. 65 (1989), 131-141.

[5] R. Bowen. Periodic points and measures for Axiom A diffeomorphism. Trans. Amer. Math. Soc. 154 (1971), 377-397.

[6] R. Bowen. Topological entropy for non-compact sets. Trans. Amer. Math. Soc. 49 (1973), 125-136.

[7] M. Brin and A. Katok. On Local Entropy. Geometric dynamics (Rio de Janeiro) (1981) (Lecture Notes in Mathematics, 1007). Springer, Berlin, 1983, pp. 30-38.

[8] C. D. Cutler. Connecting ergodicity and dimension in dynamical systems. Ergod. Th. \& Dynam. Sys. 10 (1990), 451-462.

[9] M. Denker, C. Grillenberger and K. Sigmund. Ergodic Theory on Compact Spaces (Lecture Notes in Mathematics, 527). Springer, Berlin, 1976.

[10] T. Downarowicz and B. Weiss. Entropy theorems along times when $x$ visits a set. Illinois. J. Math. 48 (2004), 59-69.

[11] G. A. Edgar and R. D. Mauldin. Multifractal decompositions of digraph recursive fractals. Proc. London Math. Soc. 65 (1992), 604-628.

[12] M. Eisenberg. Expansive transformation semigroups of automorphisms. Fund. Math. 59 (1966), 313-321.

[13] A.-H. Fan and D.-J. Feng. On the distribution of long-term time averages on symbolic space. J. Stat. Phys. 99(3-4) (2000), 813-856. 
[14] A.-H. Fan, D.-J. Feng and J. Wu. Recurrence, dimension and entropy. J. London Math. Soc. 64 (2001), 229-244.

[15] D.-J. Feng and J. Wu. The Hausdorff dimension of recurrence sets in symbolic spaces. Nonlinearity 14 (2001), 81-85.

[16] A. Katok. Lyapunov exponents, entropy and periodic orbits for diffeomorphisms. Publ. Math. de l'IHÉS. 51 (1980), 137-173.

[17] R. D. Mauldin and S. C. Williams. Hausdorff dimension in graph directed constructions. Trans. Amer. Math. Soc. 309 (1988), 811-829.

[18] P. A. P. Moran. Additive functions of intervals and Hausdorff measure. Proc. Cambridge Philos. Soc. 42 (1946), 15-23.

[19] L. Olsen. First return times: multifractal spectra and divergence points. Discrete Contin. Dyn. Syst. Ser. A 10 (2004), 635-656.

[20] D. S. Ornstein and B. Weiss. Entropy and data compression schemes. IEEE Trans. Inform. Theory 39 (1993), 78-83.

[21] Y. B. Pesin. Dimension Theory in Dynamical Systems. University of Chicago Press, Chicago, 1997.

[22] Y. B. Pesin and H. Weiss. On the dimension of deterministic and random Cantor-like sets, symbolic dynamics, and the Eckmann-Ruelle conjecture. Comm. Math. Phys. 182 (1996), 105-153.

[23] W. L. Reddy. Expanding maps on compact metric spaces. Topology Appl. 13 (1982), 327-334.

[24] I. Rosenholtz. Local expansions, derivatives, and fixed points. Fund. Math. 91 (1976), 1-4.

[25] D. Ruelle. Thermodynamic Formalism. The Mathematical Structures of Classical Equilibrium Statistical Mechanics (Encyclopedia of Mathematics and its Applications, 5). Addison-Wesley, Reading, MA, 1978.

[26] B. Saussol, S. Troubetzkoy and S. Vaienti. Recurrence, dimensions and Lyapunov exponents. J. Stat. Phys. 106 (2002), 623-634.

[27] B. Saussol and J. Wu. Recurrence spectrum in smooth dynamical system. Nonlinearity 16 (2003), 19912001.

[28] J. Schmeling. Symbolic dynamics for $\beta$-shifts and self-normal numbers. Ergod. Th. \& Dynam. Sys. 17 (1997), 675-694.

[29] L. Shu. Poincaré recurrence, measure theoretic and topological entropy. PhD Thesis, CUHK, 2007.

[30] K. Sigmund. On dynamical systems with the specification property. Trans. Amer. Math. Soc. 190 (1974), 285-289.

[31] F. Takens and E. Verbitskiy. On the variational principle for the topological entropy of certain noncompact sets. Ergod. Th. \& Dynam. Sys. 23 (2003), 317-348.

[32] P. Walters. An Introduction to Ergodic theory (Graduate Texts in Mathematics, 79). Springer, Berlin, 1982.

[33] R. F. Williams. A note on unstable homeomorphism. Proc. Amer. Math. Soc. 6 (1955), 308-309. 